\title{
Dirichlet Forms on Fractals and Products of Random Matrices
}

\author{
Dedicated to Professor Tosihusa Kimura on his 60th birthday
}

By

\section{Shigeo KusuoKA*}

\begin{abstract}
The author studies Dirichlet forms on fractals. He constructs some local Dirichlet forms on abstract fractal sets by using products of random matrices. Also, he studies the martingale dimension of the associated diffusion processes and its self-similarity.
\end{abstract}

\section{$\S 0$. Introduction}

Recently "Brownian motion" on Sierpinski gaskets were constructed by probabilistic approach (Goldstein [6], Kusuoka [9], Barlow-Parkins [1]). These are symmetric diffusion processes, and so the theory of Dirichlet forms applies to them. On the other hand, Kigami [8] introduced "Laplacian" on Sierpinski gaskets by analytic approach. Of course, these two approach reached the same object. However, any explicit expression of the associated Dirichlet forms has been unknown. In the present paper, we give their explicit expression by using products of random matrices.

In the theory of Dirichlet forms (Fukushima [5]), if a symmetric diffusion process on a locally compact space is given, one can define the associated Dirichlet forms, and moreover, one can define the signed measure $\mu^{[u, v]}$ on the state space for any elements $u, v$ in the domain of the Dirichlet form. For example, if we think of the usual Brownian motion in Euclidean space $\boldsymbol{R}^{d}$, the associated Dirichlet form is given by $\frac{1}{2} \int_{R^{d}}(\operatorname{grad} u, \operatorname{grad} v) d x$, and $\mu^{[u, v]}(d x)=$ $\frac{1}{2}(\operatorname{grad} u, \operatorname{grad} v) d x$. So we may think that to describe the Dirichlet form is to describe the signed measures $\mu^{[u, v]}$. We will focus on the explicit expression of $\mu^{[u, v]}$.

Research partially supported by Grant-in-Aid for Science Research 63740115 Min. Education.

Received January 26, 1989.

* Research Institute for Mathematical Sciences, Kyoto University, Kyoto 606, Japan. 
Since our results are quite complicated, we show only two results which follow from our results in the present paper. Let us think of the "Brownian motion" on Sierpinskii gaskets and the fractal measure $\tilde{\nu}$ on Sierpinskii gasket. Then the "Brownian motion" is $\tilde{\nu}$-symmetric.

(1) We give a measure $\tilde{\mu}$ on the Sierpinskii gasket which is singular relative to $\tilde{\nu}$, and we show that any $\mu^{[u, v]}$ is absolutely continuous relative to $\tilde{\mu}$ for any elements $u, v$ in the domain of the Dirichlet form. We also give its Radon-Nykodim density.

(2) We show that the martingale dimension is one. This answers to Problem 10.6 in Barlow-Perkins [1]. There they guessed that the dimension is $d(\geqq 2)$.

Our approach is quite abstract and we believe that our results also apply to the diffusion processes on nested fractal which were recently constructed by Lindstrøm [10]. So we discuss the relations between his results and our ones in the last of this paper.

Acknowledgement. The author thanks Prof. Y. Tamura for stimulating discussions and useful suggestions. He also thanks Professors S. Kotani, Y. Takahashi and M. Osikawa for useful discussions and informations.

\section{§1. Stationary Probability Measure}

Let $V_{0}$ be a finite dimensional real vector space with inner product (, ). We assume that the dimension of $V_{0}$ is greater than one. Let $Y_{i}, i=1, \cdots, N$, be linear operators in $V_{0}$ and $w_{i}, i=1, \cdots, N$, are positive numbers with $\sum_{i=1}^{N} w_{i}=1$.

(1.1) Definition. We say that $\left\{Y_{1}, \cdots, Y_{N}\right\}$ is irreducible if there is no vector space $V$ such that $\{0\} \subsetneq V \subsetneq V_{0}$ and $Y_{i} V \subset V$ for all $i=1, \cdots, N$.

(1.2) Theorem. Suppose that $\left\{Y_{1}, \cdots, Y_{N}\right\}$ and $\left\{{ }^{t} Y_{1}, \cdots,{ }^{t} Y_{N}\right\}$ are irreducible. Then there uniquely exist strictly positive definite symmetric linear operators $Q_{0}$ and $Q_{1}$ in $V_{0}$ and a positive number $\lambda$ such that

(1) $\operatorname{trace} Q_{0}=\operatorname{trace} Q_{1}$ and trace $Q_{0} Q_{1}=1$,

(2) $\sum_{i=1}^{N} w_{i}^{t} Y_{i} Q_{0} Y_{i}=\lambda Q_{0}$, and

(3) $\sum_{i=1}^{N} w_{i} Y_{i} Q_{1}{ }^{t} Y_{i}=\lambda Q_{1}$.

Proof. Step 1. We shall show that there are unique $\lambda>0$ and a strictly positive definite symmetric linear operator $Q$ in $V_{0}$ such that

$$
\operatorname{trace} Q=1 \text { and } \sum_{i=1}^{N} w_{i}{ }^{t} Y_{i} Q Y_{i}=\lambda Q \text {. }
$$

Let $S$ be the set of all nonnegative definite symmetric linear operators whose trace is equal to one. Note that $\sum_{i=1}^{N} w_{i}{ }^{t} Y_{i} Q Y_{i} \neq 0$ for any $Q \in S$. In fact, 
if $\sum_{\imath=1}^{N} w_{i}{ }^{t} Y_{\imath} Q Y_{\imath}=0$, then letting $V=\left\{v \in V_{0} ;(v, Q v)=0\right\}$, we have $Y_{\imath} V \subset V, i=$ $1, \cdots, N$. Since $V \neq V_{0}, V$ should be $\{0\}$ from the irreducibility. But then we see that $Y_{1}=Y_{2}=\cdots=Y_{N}=0$. This contradicts the irreducibility and the assumption that $\operatorname{dim} V_{0} \geqq 2$.

Therefore we can define a map $F: S \rightarrow S$ by $F(Q)=\left(\operatorname{trace}\left(\sum_{i=1}^{N} w_{i}{ }^{t} Y_{\imath} Q Y_{i}\right)\right)^{-1}$ $\times \sum_{i=1}^{N} w_{i}{ }^{t} Y_{i} Q Y_{i}$. Since $S$ is a compact convex space, there is a $Q \in S$ such that $F(Q)=Q$. Suppose that $F(Q)=Q$. Let $V=\{v \in V ;(v, Q v)=0\}$. Then since $\sum_{i=1}^{N} w_{\imath}\left(Y_{i} v, Q Y_{i} v\right)=(v, Q v)$, we see that $Y_{i} V \subset V, i=1, \cdots, N$. As $V \neq V_{0}$, we have $V=\{0\}$. Thus we see that $Q$ is strictly positive definite if $F(Q)=Q$.

Therefore we see that there is a strictly positive definite operator $Q$ in $S$ with $F(Q)=Q$. Letting $\lambda=\operatorname{trace} \sum_{i=1}^{N} w_{\imath}{ }^{t} Y_{i} Q Y_{i}$, we see that there are $\lambda>0$ and a strictly positive definite symmetric operator $Q$ satisfying (1.3).

Now suppose that there is another pair $\left\{\lambda^{\prime}, Q^{\prime}\right\}$ satisfying (1.3). Let $\alpha=\sup \left\{a \geqq 0 ; Q^{\prime}-a Q\right.$ is nonnegative definite $\}$. Then $Q^{\prime}-\alpha Q$ is nonnegative definite symmetric linear operator in $V_{0}$ and is degenerate, but is not equal to zero.

Then we have $1-\alpha=\operatorname{trace}\left(Q^{\prime}-\alpha Q\right)>0$ and $\sum_{i=1}^{N} w_{i}{ }^{t} Y_{i}\left(Q^{\prime}-\alpha Q\right) Y_{i}=\lambda^{\prime} Q^{\prime}-\alpha \lambda Q$. Since the right hand side of the second term is nonnegative definite we see that $\lambda^{\prime} \geqq \lambda$. Similarly we have $\lambda \geqq \lambda^{\prime}$. So $\lambda=\lambda^{\prime}$. Then we see that if we let $\tilde{Q}=\left(\operatorname{trace}\left(Q^{\prime}-\alpha Q\right)\right)^{-1}\left(Q^{\prime}-\alpha Q\right) \in S$, then $F(\tilde{Q})=\tilde{Q}$. However, $Q$ is degenerate and this is the contradiction. Therefore, a pair $\{\lambda, Q\}$ satisfying (1.3) is unique.

Step 2. From the result in Step 1 we see that there exist uniquely $Q_{0}^{\prime}, Q_{1}^{\prime} \in S$ and $\lambda_{0}, \lambda_{1}>0$ such that $\sum_{i=1}^{N} w_{\imath}^{t} Y_{i} Q_{0}^{\prime} Y_{\imath}=\lambda_{0} Q_{0}^{\prime}$ and $\sum_{i=1}^{N} w_{\imath} Y_{\imath} Q_{1}^{\prime}{ }^{t} Y_{i}=\lambda_{1} Q_{1}^{\prime}$. Then we have

$$
\begin{aligned}
\lambda_{0} \operatorname{trace}\left(Q_{0}^{\prime} Q_{1}^{\prime}\right) & =\operatorname{trace}\left(\left(\sum_{\imath=1}^{N} w_{\imath}{ }^{t} Y_{i} Q_{0}^{\prime} Y_{\imath}\right) Q_{1}^{\prime}\right) \\
& =\operatorname{trace}\left(Q_{0}^{\prime}\left(\sum_{\imath=1}^{N} w_{\imath} Y_{\imath} Q_{1}^{t} Y_{\imath}\right)\right)=\lambda_{1} \operatorname{trace}\left(Q_{0}^{\prime} Q_{1}^{\prime}\right) .
\end{aligned}
$$

This implies $\lambda_{0}=\lambda_{1}$. Thus letting $Q_{\imath}=\left(\operatorname{trace} Q_{0}^{\prime} Q_{1}^{\prime}\right)^{-1 / 2} Q_{\imath}^{\prime}, i=0,1$, and $\lambda=\lambda_{0}=\lambda_{1}$, we have our assertion.

Q.E.D.

In this section and the next section, we always assume that $\left\{Y_{1}, \cdots, Y_{N}\right\}$ and $\left\{{ }^{t} Y_{1}, \cdots,{ }^{t} Y_{N}\right\}$ are irreducible. So we have strictly positive definite symmetric linear operators $Q_{0}$ and $Q_{1}$ and a positive number $\lambda>0$ as in Theorem (1.2).

(1.4) Definition. We say that a probability measure $\mu$ in $\Omega=\{1, \cdots, N\}^{N}$ is associated to $\left(\left\{Y_{1}, \cdots, Y_{N}\right\},\left\{w_{1}, \cdots, w_{N}\right\}\right)$ if 


$$
\begin{aligned}
& \mu\left(\left\{\omega \in \Omega ; \omega_{1}=i_{1}, \omega_{2}=i_{2}, \cdots, \omega_{n}=i_{n}\right\}\right) \\
& =\lambda^{-n} w_{i_{1}} w_{i_{2}} \cdots w_{i_{n}} \cdot \operatorname{trace}\left(Q_{1}^{t} Y_{i_{1}}{ }^{t} Y_{i_{2}}{ }^{t}{ }^{t} Y_{i_{n}} Q_{0} Y_{i_{n}} Y_{i_{n-1}} \cdots Y_{i_{1}}\right)
\end{aligned}
$$

for any $i_{1}, i_{2}, \cdots, i_{n} \in\{1, \cdots, N\}$.

(1.6) Remark. By virtue of Theorem (1.2), (1.5) satisfies the consistence condition. Therefore there is a unique probability measure $\mu$ in $\Omega$ associated to $\left(\left\{Y_{1}, \cdots, Y_{N}\right\},\left\{w_{1}, \cdots, w_{N}\right\}\right)$. By (1.5), we see that the measure $\mu$ is stationary, i. e., $\mu \circ T^{-1}=\mu$, where $T$ is a map from $\Omega$ onto $\Omega$ given by $(T \omega)_{n}=\omega_{n+1}, n \in N$.

In this section and the next section, $\mu$ always denotes the probability measure associated to $\left(\left\{Y_{1}, \cdots, Y_{N}\right\},\left\{w_{1}, \cdots, w_{N}\right\}\right)$ and $\nu$ be a Bernoulli measure in $\Omega$ with $\nu\left(\omega_{1}=i\right)=w_{i}, i \in\{1, \cdots, N\}$. Let $\mathscr{F}_{n}^{m}, 1 \leqq n \leqq m \leqq \infty$, be a $\sigma$-algebra in $\Omega$ generated by $\left\{\omega_{i} ; n \leqq i<m+1\right\}$. Let $X_{n}(\omega)=Y_{\omega_{n}}, n \in N$ and $\omega \in \Omega$, and let $W_{n}(\omega)=X_{n}(\omega) X_{n-1}(\omega) \cdots X_{1}(\omega), \quad n \geqq 1$ and $\omega \in \Omega$. Finally, let $Z_{n}(\omega)=$ $\left(\operatorname{trace}\left(Q_{1}{ }^{t} W_{n}(\omega) Q_{0} W_{n}(\omega)\right)\right)^{-1}{ }^{t} W_{n}(\omega) Q_{0} W_{n}(\omega), n \geqq 1$.

(1.7) Proposition. (1) $Z_{n}(\omega)$ is defined $\mu$-a.e. $\omega$ and trace $\left(Q_{1} Z_{n}(\omega)\right)=1$, $\mu$-a.s. $\omega$.

(2) $\left\{Z_{n}(\omega), \mathscr{I}_{1}^{n}, n \geqq 1\right\}$ is a matringale under $\mu$. Therefore $Z(\omega)=\lim _{n \rightarrow \infty} Z_{n}(\omega)$ exists $\mu$-a.s. $\omega$.

(3) $Z(\omega)=\left(\operatorname{trace} Q_{1}{ }^{t} X_{1}(\omega) Z(T \omega) X_{1}(\omega)\right)^{-1}{ }^{t} X_{1}(\omega) Z(T \omega) X_{1}(\omega), \mu-a . s . \omega$.

(4) For any $n \geqq 1$ and $f \in C\left(\{1, \cdots, N\}^{n} ; \mathbb{R}\right)$,

$$
\begin{aligned}
& E^{\mu}\left[f\left(X_{1}, \cdots, X_{n}\right) \mid \mathscr{F}_{n+1}^{\infty}\right](\omega) \\
= & \lambda^{-n} \int_{\Omega} f\left(X_{1}(\widetilde{\omega}), \cdots, X_{n}(\widetilde{\omega})\right) \cdot \operatorname{trace}\left(Q_{1}{ }^{t} W_{n}(\widetilde{\omega}) Z\left(T^{n} \omega\right)^{t} W_{n}(\widetilde{\omega})\right) \nu(d \widetilde{\omega})
\end{aligned}
$$

for $\mu$-a.s. $\omega$.

Proof. (1) is obvious, since $\mu\left(\operatorname{trace}\left(Q_{1}{ }^{t} W_{n}(\omega) Q_{0} W_{n}(\omega)\right)=1\right)=1$. (2) comes from the following.

$$
\begin{aligned}
& E^{\mu}\left[Z_{n+1} \mid \mathscr{F}_{1}^{n}\right](\omega) \\
= & \left(\lambda^{-n} \cdot \operatorname{trace}\left(Q_{1}{ }^{t} W_{n}(\omega) Q_{0} W_{n}(\omega)\right)\right)^{-1} \cdot E^{\nu}\left[\left(\lambda^{n+1} \operatorname{trace}\left(Q_{1}{ }^{t} W_{n+1} Q_{0} W_{n+1}\right)\right) Z_{n+1} \mid \mathscr{I}_{1}^{n}\right] \\
= & \left(\operatorname{trace}\left(Q_{1}{ }^{t} W_{n}(\omega) Q_{0} W_{n}(\omega)\right)\right)^{-1} \sum_{i=1}^{N} \lambda^{t} W_{n}(\omega)^{t} Y_{2} Q_{0} Y_{i} W_{n}(\omega) \\
= & Z_{n}(\omega) .
\end{aligned}
$$

Then we have

$$
\begin{aligned}
& Z(\omega)=\lim _{n \rightarrow \infty} Z_{n+1}(\omega) \\
= & \lim _{n \rightarrow \infty}\left(\operatorname{trace} Q_{1}{ }^{t} X_{1}(\omega) Z_{n}(T \omega) X_{1}(\omega)\right)^{-1}{ }^{t} X_{1}(\omega) Z_{n}(T \omega) X_{1}(\omega) .
\end{aligned}
$$

This implies the assertion (3). Also, we have 


$$
\begin{aligned}
& E^{\mu}\left[f\left(X_{1}, \cdots, X_{n}\right) \mid \mathscr{T}_{n+1}^{\infty}\right] \\
= & \lim _{m \rightarrow \infty} E^{\mu}\left[f\left(X_{1}, \cdots, X_{n}\right) \mid \mathscr{F}_{n+1}^{n+m}\right] \\
= & \lim _{m \rightarrow \infty} \sum_{i_{1}, \cdots, i_{n}=1}^{N} \lambda^{-n} f\left(i_{1}, \cdots, i_{n}\right) w_{i_{1}} \cdots w_{i_{n}} \\
& \quad \times \operatorname{trace}\left(Q_{1}{ }^{t} Y_{i_{1}} \cdots{ }^{t} Y_{i_{n}} Z_{m}\left(T^{n} \omega\right) Y_{i_{n}} \cdots Y_{i_{1}}\right) .
\end{aligned}
$$

This implies the assertion (4).

(1.8) Proposition. Let $a(p)=\inf _{n} \lambda^{-n} \cdot E^{\nu}\left[\left\|\stackrel{p}{\wedge} W_{n}\right\|_{\wedge V_{0} \rightarrow \wedge V_{0}}^{p}{ }^{2 / p}\right], p=1, \cdots, \operatorname{dim} V_{0}$. Then we have the following.

(1) $a(p) \geqq 1$ or $a(p)=0$.

(2) There are $c_{0}, c_{1} \in(0, \infty)$ for each $p=1, \cdots, \operatorname{dim} V_{0}$, such that

$$
c_{0} a(p) \leqq E^{\mu}\left[\left\|\wedge{ }^{p} Z\right\|_{\wedge V_{0} \rightarrow \wedge V_{0}}^{p}{ }^{1 / p}\right] \leqq c_{1} a(p) .
$$

Proof. Let $a_{n}(p)=\lambda^{-n} \cdot E^{\nu}\left[\left\|\wedge W_{n}\right\|_{\wedge V_{0} \rightarrow \wedge V_{0}}^{p / p}\right]$. Then we have

$$
\begin{aligned}
a_{n+m}(p) & \leqq \lambda^{-n-m} \cdot E^{\mu}\left[\left\|\wedge W_{n}(\omega)\right\|_{\stackrel{p}{\wedge V_{0} \rightarrow \wedge V_{0}}}{ }^{2 / p}\left\|\stackrel{p}{\wedge} W_{m}\left(T^{n} \omega\right)\right\|_{\wedge V_{0} \rightarrow \wedge V_{0}}{ }^{2 / p}\right] \\
& \leqq a_{n}(p) a_{m}(p) .
\end{aligned}
$$

Therefore we see that if $a(p)<1$, then $a(p)=0$.

Note that

and

$$
E^{\mu}\left[\left\|\stackrel{p}{\wedge} Z_{n}\right\|_{\wedge V_{0} \rightarrow \wedge V_{0}}^{p}{ }^{1 / p}\right]=\left(\operatorname{trace}\left(Q_{1} Q_{0}\right)\right)^{-1} \lambda^{-n} \cdot E^{\nu}\left[\left\|\wedge^{p}\left({ }^{t} W_{n} Q_{0} W_{n}\right)\right\|_{\wedge V_{0} \rightarrow \wedge V_{0}}^{p}{ }^{1 / p}\right]
$$

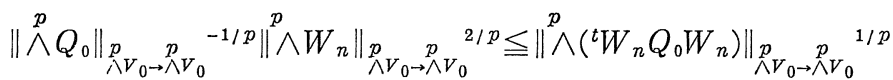

$$
\begin{aligned}
& \leqq\left\|\stackrel{p}{\wedge} Q_{0}\right\|_{\wedge V_{0} \rightarrow \wedge V_{0}}^{p}{ }^{1 / p}\left\|\stackrel{p}{\wedge} W_{n}\right\|_{\wedge V_{0} \rightarrow \wedge V_{0}}^{p}{ }^{2 / p}
\end{aligned}
$$

This implies our assertions.

(1.10) Definition. We call $p_{0}=\max \{p \geqq 1 ; a(p) \geqq 1\}$ the index of $\left(\left\{Y_{1}, \cdots, Y_{N}\right\}\right.$, $\left.\left\{w_{1}, \cdots, w_{N}\right\}\right)$.

(1.11) Corollary. Let $p_{0}$ be the index. Then $\mu\left(\operatorname{rank} Z(\omega) \leqq p_{0}\right)=1$ and $\mu(\operatorname{rank}$ $\left.Z(\omega)=p_{0}\right)>0$.

\section{§. The Related Markov Chain}

We use the notation in Section 1. In this section we assume that each $Y_{i}$, $i=1, \cdots, N$, is invertible and $\left\{Y_{1}, \cdots, Y_{N}\right\}$ is irreducible. Then $\left\{{ }^{t} Y_{1}, \cdots,{ }^{t} Y_{N}\right\}$ is also irreducible.

Let $S$ be the set of nonnegative definite symmetric linear operators $A$ in $V_{0}$ with trace $Q_{1} A=1$. We let 


$$
\begin{aligned}
& p(A, i)=\lambda^{-1} w_{i} \text { trace } Q_{1}{ }^{t} Y_{i} A Y_{i} \text { and } \\
& B(A, i)=\left(\operatorname{trace} Q_{1}{ }^{t} Y_{i} A Y_{i}\right)^{-1} \cdot{ }^{t} Y_{i} A Y_{i}
\end{aligned}
$$

for each $A \in S$ and $i \in\{1, \cdots, N\}$. Then $B(\cdot, \cdot)$ is a map from $S \times\{1, \cdots, N\}$ into $S$ and

$$
\sum_{i=1}^{N} p(A, i)=1, \quad A \in S \text {. }
$$

Let us define a probability measure $P((i, A), \cdot)$ in $\tilde{S}=\{1, \cdots, N\} \times S$ by

$$
P((i, A), E)=\sum_{j=1}^{N} p(A, j) \cdot \chi_{E}((j, B(A, j))), \quad E \in \mathscr{B}(\tilde{S})
$$

for any $(i, A) \in \widetilde{S}$. Then this $P$ define a Markov chain in $\widetilde{S}$. Let $\left\{P_{z} ; z \in \widetilde{S}\right\}$ be a family of probability measure in $\Theta=\tilde{S}^{(0) \cup N}$ which defines the Markov chain associated $P(z, \cdot)$, i.e.,

$$
\begin{aligned}
& P_{z}\left[\left\{\theta \in \Theta ; \theta_{0} \in E_{0}, \theta_{1} \in E_{1}, \cdots, \theta_{n} \in E_{n}\right\}\right] \\
= & \chi_{E_{0}}(z) \cdot \int_{E_{1} \times \cdots \times E_{n}} P\left(z, d z_{1}\right) P\left(z_{1}, d z_{2}\right) \cdots P\left(z_{n-1}, d z_{n}\right)
\end{aligned}
$$

for any $z \in \widetilde{S}, E_{0}, E_{1}, \cdots, E_{n} \in \mathscr{B}(\tilde{S})$.

Let $m_{0}$ be the probability distribution in $\tilde{S}$ induced by $\left(\omega_{1}, Z(\omega)\right)^{*}$ under $\mu$.

(2.5) Proposition. $m_{0}$ is P-invariant, i.e.,

$$
m_{0} P(E) \underset{\text { def }}{=} \int_{\tilde{S}} m_{0}(d z) P(z, E)=m_{0}(E) .
$$

Proof. By Proposition (1.7)(3), we have $B\left(Z(T \omega), \omega_{1}\right)=Z(\omega), \mu$-a.s. $\omega$. Therefore for any $f \in C(\tilde{S} ; \boldsymbol{R})$,

$$
\begin{aligned}
\int_{\tilde{S}} f(z) m_{0}(d z) & =E^{\mu}\left[E^{\mu}\left[f\left(\omega_{1}, B\left(Z(T \omega), \omega_{1}\right)\right) \mid \mathscr{I}_{2}^{\infty}\right]\right] \\
& =E^{\mu}\left[\sum_{i=1}^{N} p(Z(T \omega), i) f(i, B(Z(T \omega), i))\right] \\
& =\int_{\tilde{S}} m_{0}\left(d z_{1}\right) \int_{\tilde{S}} f\left(z_{2}\right) P\left(z_{1}, d z_{2}\right) .
\end{aligned}
$$

This proves our assertion.

Let $p r_{S}$ denote the natural projection map from $\tilde{S}$ into $S$. Let $S_{p}=$ $\{A \in S$; the rank of $A$ is $p\}, p=1, \cdots, \operatorname{dim} V_{0}$ and let $\widetilde{S}_{p}=\operatorname{pr}_{s}^{-1}\left(S_{p}\right)$. Let $M$ denote the set of all $P$-invariant probability measure $m$ in $\tilde{S}$ such that $P_{m}$ is ergodic. Since $P\left(z, \widetilde{S}_{p}\right)=1$ if $z \in \widetilde{S}_{p}$, we see that there is a $p \in\left\{1, \cdots, \operatorname{dim} V_{0}\right\}$ for each $m \in M$ such that $m\left(\tilde{S}_{p}\right)=1$.

(2.6) Proposition. For any $m \in M,\left\{v \in V_{0} ;(v, A v)=0\right.$ for all $\left.A \in p r_{S}(\operatorname{supp}(m))\right\}$ $=\{0\}$. 
Proof. Let $V=\left\{v \in V_{0} ;(v, A v)=0\right.$ for all $\left.A \in p r_{s}(\operatorname{supp}(m))\right\}$. If $(i, A) \in \operatorname{supp}(m)$, then $(j, B(A, j)) \in \operatorname{supp}(m), j=1, \cdots, N$. Therefore we see that $\left(v,{ }^{t} Y_{j} A Y_{j} v\right)=0$, $j=1, \cdots, N$, for any $v \in V$ and $A \in \operatorname{pr}_{s}(\operatorname{supp}(m))$. So we have $Y_{i} V \subset V, i=1, \cdots, N$. Since $V \neq V_{0}, V=\{0\}$. This completes the proof.

(2.7) Proposition. Let $Z_{n}^{A}(\boldsymbol{\omega})=\left(\operatorname{trace} Q_{1}{ }^{t} W_{n}(\boldsymbol{\omega}) A W_{n}(\boldsymbol{\omega})\right)^{-1}{ }^{t} W_{n}(\boldsymbol{\omega}) A W_{n}(\boldsymbol{\omega})$ for any $A \in S$ and $\omega \in \Omega$. Then we have

$$
\begin{aligned}
& P_{(i, A)}\left[\operatorname{pr}_{s}\left(\theta_{0}\right) \in C_{0}, \cdots, \operatorname{pr}_{S}\left(\theta_{n}\right) \in C_{n}\right] \\
&= E^{\mu}\left[\left(\operatorname{trace} Q_{1}{ }^{t} W_{n} A W_{n}\right)\left(\operatorname{trace} Q_{1}{ }^{t} W_{n}(\omega) Q_{0} W_{n}(\omega)\right)^{-1}, Z_{k}^{A}\left(T^{n-k} \omega\right) \in C_{k},\right. \\
&k=0,1, \cdots, n]
\end{aligned}
$$

for any $n \geqq 1$ and $C_{0}, \cdots, C_{n} \in \mathscr{B}(S)$.

Proof. This comes from the following.

$$
\begin{aligned}
& P_{(i, A)}\left[p r_{S}\left(\theta_{0}\right) \in C_{0}, \cdots, p r_{S}\left(\theta_{n}\right) \in C_{n}\right] \\
&= \sum_{i_{1}, \cdots, i_{n}=1}^{N} \lambda^{-n} w_{i_{1}} \cdots w_{i_{n}} \operatorname{trace} Q_{1}{ }^{t} Y_{i_{n}} \cdots{ }^{t} Y_{i_{1}} A Y_{i_{1}} \cdots Y_{i_{n}} \\
& \cdot \chi_{C_{0}}(A) \chi_{C_{1}}\left(B\left(A, i_{1}\right)\right) \cdots \chi_{C_{n}}\left(B\left(\cdots B\left(B\left(A, i_{1}\right), i_{2}\right), \cdots, i_{n}\right)\right. \\
&= \sum_{i_{1}, \cdots, i_{n}=1}^{N} \lambda^{-n} w_{i_{1}} \cdots w_{i_{n}} \operatorname{trace} Q_{1}{ }^{t} Y_{i_{1}} \cdots{ }^{t} Y_{i_{n}} A Y_{i_{n}} \cdots Y_{i_{1}} \\
& \cdot \chi_{C_{0}}(A) \chi_{C_{1}}\left(B\left(A, i_{n}\right)\right) \cdots \chi_{C_{n}}\left(B\left(\cdots B\left(B\left(A, i_{n}\right), i_{n-1}\right), \cdots, i_{1}\right)\right. \\
&=\lambda^{-n} E^{\nu}\left[\operatorname{trace} Q_{1}{ }^{t} W_{n}(\omega) A W_{n}(\omega), Z_{k}^{A}\left(T^{n-k} \omega\right) \in C_{k} \quad \text { for } k=0, \cdots, n\right] \\
&= E^{\mu}\left[\left(\operatorname{trace} Q_{1}{ }^{t} W_{n} A W_{n}\right)\left(\operatorname{trace} Q_{1}{ }^{t} W_{n}(\omega) Q_{0} W_{n}(\omega)\right)^{-1}, Z_{k}^{A}\left(T^{n-k} \omega\right) \in C_{k}\right.
\end{aligned}
$$

Q.E.D.

(2.8) Theorem. Let $p_{0}$ be the index of $\left(\left\{Y_{1}, \cdots, Y_{N}\right\},\left\{w_{1}, \cdots, w_{N}\right\}\right)$. Then

$$
\begin{aligned}
& \mu\left[\operatorname{rank} Z(\boldsymbol{\omega})=p_{0}\right]=1 \text { and } \\
& p_{0}=\max \left\{p \in\left\{1, \cdots, \operatorname{dim} V_{0}\right\} ; m\left(\tilde{S}_{p}\right)=1 \text { for some } m \in M\right\} .
\end{aligned}
$$

Proof. Since $m_{0}\left(\widetilde{S}_{p_{0}}\right)>0$ by Corollary (1.11), we see that there is an $m \in M$ such that $m\left(\tilde{S}_{p_{0}}\right)=1$. Let $q=\max \left\{p \in\left\{1, \cdots, \operatorname{dim} V_{0}\right\} ; m\left(\widetilde{S}_{p}\right)=1\right.$ for some $\left.m \in M\right\}$ and take an $m \in M$ such that $m\left(\widetilde{S}_{q}\right)=1$. Then $q \geqq p_{0}$.

Since $P_{m}$ is ergodic, we see that

$$
P_{z}\left[\frac{1}{n} \sum_{k=1}^{n} f\left(\theta_{k}\right) \rightarrow \int_{\tilde{S}} f d m \text { for all } f \in C(\tilde{S} ; \boldsymbol{R})\right]=1, \quad m \text {-a. e. } z .
$$

Therefore, by Proposition (2.6), we see that there are $\left(i_{j}, A_{j}\right) \in \widetilde{S}, j=1, \cdots, d$, such that $\sum_{j=1}^{d} A_{j}$ is strictly positive definite and 


$$
\left.P_{\left(i_{j}, A\right.}\right)\left[\frac{1}{n} \sum_{k=1}^{n} f\left(\boldsymbol{\theta}_{k}\right) \rightarrow \int_{\tilde{S}} f d m \text { for all } f \in C(\tilde{S} ; \boldsymbol{R})\right]=1
$$

for $j=1, \cdots, d$. Therefore

$$
\lim _{n \rightarrow \infty} \frac{1}{n} \sum_{k=1}^{n} E^{P}\left(i_{\jmath}, A_{\jmath}\right)\left[f\left(\theta_{k}\right)\right]=\int_{\tilde{S}} f d m
$$

for any $f \in C(\widetilde{S} ; \boldsymbol{R})$ and $j=1, \cdots, d$.

Take a $\delta>0$ such that $\delta \cdot Q_{0} \leqq \sum_{j=1}^{d} A_{j} \leqq \delta^{-1} Q_{0}$. Let

$$
\rho_{j, n}(\boldsymbol{\omega})=\left(\operatorname{trace} Q_{1}{ }^{t} W_{n}(\boldsymbol{\omega}) A_{\jmath} W_{n}(\boldsymbol{\omega})\right) \cdot\left(\operatorname{trace} Q_{1}{ }^{t} W_{n}(\boldsymbol{\omega}) Q_{0} W_{n}(\boldsymbol{\omega})\right)^{-1}, \quad n \geqq 1
$$

and $j=1, \cdots, d$. Then we have $Z_{n}{ }^{A}{ }(\omega) \leqq\left(\delta \cdot \rho_{j, n}(\omega)\right)^{-1} \cdot Z_{n}(\omega)$ and $\sum_{j=1}^{d} \rho_{j, n}(\omega) \geqq \delta$. Therefore we have for any $\varepsilon>0$

$$
\begin{aligned}
& \mu\left[\left\|\stackrel{q}{\wedge} Z_{n}\right\|_{\wedge V_{0} \rightarrow \wedge V_{0}}<\varepsilon\right] \\
& \leqq \sum_{j=1}^{d} \mu\left[\left\|\stackrel{q}{\wedge} Z_{n}{ }^{A}\right\|_{\wedge V_{0} \rightarrow \wedge V_{0}}^{q} \leqq d^{q} \delta^{-2 q} \varepsilon, \rho_{j, n} \geqq d^{-1} \delta\right] \\
& \leqq d \cdot \delta^{-1} \cdot \sum_{j=1}^{d} E^{\mu}\left[\rho_{j, n},\left\|\stackrel{q}{\wedge} Z_{n}{ }^{A}\right\|_{\wedge V_{0 \rightarrow} \rightarrow \wedge V_{0}} \leqq d^{q} \delta^{-2 q} \varepsilon\right] \\
& \leqq d \cdot \delta^{-1} \cdot \sum_{j=1}^{d} P_{\left(i_{\jmath}, A_{\jmath}\right)}\left[\left\|\wedge \wedge^{q}\left(p r_{S}\left(\theta_{n}\right)\right)\right\|_{\wedge V_{0} \rightarrow \wedge V_{0}} \leqq d^{q} \delta^{-2 q} \varepsilon\right] .
\end{aligned}
$$

Therefore we have

$$
\begin{aligned}
& \mu\left[\|\stackrel{q}{\wedge} Z\|_{\wedge q} \underset{\wedge V_{0} \rightarrow \wedge V_{0}}{q}<\varepsilon\right] \\
\leqq & \varlimsup_{n \rightarrow \infty} \frac{1}{n} \sum_{k=1}^{n} \mu\left[\left\|\stackrel{q}{\wedge} Z_{k}\right\|_{\wedge V_{0} \rightarrow \wedge^{q} V_{0}}<\varepsilon\right] \\
\leqq & d \cdot \delta^{-1} \cdot \sum_{j=1}^{d} \varlimsup_{n \rightarrow \infty} \frac{1}{n} \sum_{k=1}^{n} P_{\left(i_{j}, A_{j}\right.}\left[\left\|\wedge\left(p r_{S}\left(\theta_{k}\right)\right)\right\|_{\wedge V_{0} \rightarrow \wedge V_{0}} \leqq d^{q} \delta^{-2 q} \varepsilon\right] . \\
\leqq & d^{2} \delta^{-1} \cdot m\left(\left\{z \in \widetilde{S} ;\left\|\wedge\left(p r_{S}(z)\right)\right\|_{\wedge}^{q} \underset{\wedge V_{0} \rightarrow \wedge V_{0}}{q} \leqq d^{q} \delta^{-2 q} \varepsilon\right\}\right) \\
\rightarrow & 0 \quad \text { as } \varepsilon \downarrow 0 .
\end{aligned}
$$

This proves that $\mu[\operatorname{rank} Z(\omega) \geqq q]=1$. This implies our assertions.

Let $G_{p}\left(V_{0}\right), p=1, \cdots, \operatorname{dim} V_{0}$, be the set of all $p$-dimensional vector subspace of $V_{0}$. Then $G_{p}$ is a compact manifold. Let $\sigma_{p}$ be a map from $S_{p}$ into $G_{p}$ defined by $\sigma_{p}(A)=\left\{A v \in V_{0} ; v \in V_{0}\right\}, A \in S_{p}$. Then $\sigma_{p}$ is a continuous map. Let $\tilde{\sigma}_{p}=\sigma_{p} \circ p r_{S}: \widetilde{S}_{p} \rightarrow G_{p}$ 。

(2.11) Theorem. Let $q$ be the index. Then $m_{0}^{\circ} \tilde{\sigma}_{q}^{-1}$ is absolutely continuous relative to $m \circ \tilde{\sigma}_{q}^{-1}$ for any $m \in M$ with $m\left(\widetilde{S}_{q}\right)=1$. Moreover, the Radon-Nykodim density is bounded. 
Proof. For any compact set $K$ in $G_{q}$ and $\varepsilon, \gamma>0$, let $\bar{K}_{\varepsilon, \gamma}$ be a set given by $\bar{K}_{\varepsilon, \gamma}=\left\{A \in S_{q} ;\right.$ there are $A^{\prime} \in S_{q}$ and $B \in S$ such that $\sigma_{q}\left(A^{\prime}\right) \in K$,

$$
\left.\left\|\stackrel{q}{\wedge} A^{\prime}\right\|_{\wedge \underset{\wedge}{\wedge} V_{0} \rightarrow \wedge V_{0}} \geqq \gamma,\left\|A^{\prime}-B\right\|_{V_{0} \rightarrow V_{0}} \leqq \varepsilon \text { and } B \geqq \gamma A\right\} .
$$

Then $\bar{K}_{\varepsilon, \gamma}$ is a compact set in $S$ and $\bigcap_{\varepsilon>0} \sigma_{q}\left(\bar{K}_{\varepsilon, \gamma}\right) \subset K$ for any $\gamma>0$.

Let us use the notation in the proof of Theorem (2.8). Take a $\gamma>0$ with $\gamma<d^{-1} \delta^{2}$. Then we have

$$
\begin{aligned}
& m\left(\operatorname{pr}_{\bar{S}}^{-1}\left(\bar{K}_{\varepsilon, \gamma}\right)\right) \geqq \frac{1}{d} \sum_{j=1}^{d} \varlimsup_{n \rightarrow \infty} \frac{1}{n} \sum_{k=1}^{n} P_{\left(i_{j}, A_{j}\right)}\left[\theta_{k} \in \operatorname{pr}^{-1}\left(\bar{K}_{\varepsilon, \gamma}\right)\right] \\
& \geqq \frac{1}{d} \varlimsup_{n \rightarrow \infty} \frac{1}{n} \sum_{j=1}^{d} \sum_{k=1}^{n} E^{\mu}\left[\rho_{j, k}, Z_{k}{ }^{\Lambda_{j}} \in \bar{K}_{\varepsilon, \gamma}\right] \\
& \geqq \frac{1}{d} \varlimsup_{n \rightarrow \infty} \frac{1}{n} \sum_{j=1}^{d} \sum_{k=1}^{n} d^{-1} \delta \cdot \mu\left[\rho_{j, k} \geqq d^{-1} \delta,\left\|Z-Z_{k}\right\|_{V_{0} \rightarrow V_{0}} \leqq \varepsilon,\right. \\
& \left.\|\stackrel{q}{\wedge} Z\|_{\wedge V_{0} \rightarrow \wedge V_{0}} \geq \gamma, \sigma_{q}(Z) \in K\right] \\
& \geqq d^{-2} \delta \cdot \varlimsup_{n \rightarrow \infty} \frac{1}{n} \sum_{k=1}^{n} \mu\left[\left\|Z-Z_{k}\right\|_{V_{0} \rightarrow V_{0}} \leqq \varepsilon,\|\stackrel{q}{\wedge} Z\|_{\wedge \underset{\wedge}{\wedge V_{0} \rightarrow \wedge V_{0}}} \geqq \gamma, \sigma_{q}(Z) \in K\right] \\
& =d^{-2} \delta \cdot \mu\left[\|\stackrel{q}{\wedge} Z\|_{\wedge V_{0} \rightarrow \wedge V_{0}} \geqq \gamma, \sigma_{q}(Z) \in K\right] .
\end{aligned}
$$

Letting $\varepsilon \downarrow 0$ first and letting $\gamma \downarrow 0$, we have $m_{0} \circ \tilde{\sigma}_{q}^{-1}(K) \leqq d^{2} \delta^{-1} m \circ \tilde{\sigma}_{q}^{-1}(K)$ for any compact set $K$ in $G_{q}$. This implies our assertion.

(2.12) Corollary. If the index equals one, then $\#(M)=1$ and $\mu$ is ergodic.

Proof. Note that $\sigma_{1}: S_{1} \rightarrow G_{1}\left(V_{0}\right)$ is one-to-one. Therefore we see that $m_{0} \circ p_{S}^{-1}$ is absolutely continuous relative to $m \circ p r_{S}^{-1}$ for any $m \in M$. Since $P((i, A), E), E \in \mathscr{B}(\tilde{S})$ is independent of $i$, we see that $m_{0}=m_{0} P$ is absolutely continuous relative to $m=m P$. Therefore $P_{m_{0}}$ is absolutely continuous relative to $P_{m}$. But this implies that $m=m_{0}$. This completes the proof.

(2.13) Definition. We say that $\left\{Y_{1}, \cdots, Y_{N}\right\}$ is strongly irreducible, if there does not exist a finite family of proper linear subspaces of $V_{0}, V_{1}, \cdots, V_{k}$ such that

$$
Y_{\imath}\left(V_{1} \cup V_{2} \cdots \cup V_{k}\right)=V_{1} \cup V_{2} \cdots \cup V_{k}
$$

for all $i=1, \cdots, N$.

(2.14) Theorem. Let $T$ be the semigroup in $G L\left(V_{0}\right)$ generated by $\left\{Y_{1}, \cdots, Y_{N}\right\}$ $\subset G L\left(V_{0}\right)$. Suppose that $\left\{Y_{1}, \cdots, Y_{N}\right\}$ is strongly irreducible and there is a sequence $\left\{M_{n}\right\}_{n=1}^{\infty}$ in $T$ such that $\left\|M_{n}\right\|^{-1} M_{n}$ converges to a matrix whose rank is less than the index $p$. Then the probability measures $\nu$ and $\mu$ are mutually singular. 
Proof. Suppose that $\nu$ and $\mu$ are not mutually singular. Since $\nu$ is ergodic, $\nu$ is absolutely continuous relative to $\mu$. Thus by Proposition $(1.7)$, we see that for $\nu$-a.s. $\omega,\left\|{ }^{t} W_{n}(\omega) Q_{0} W_{n}(\omega)\right\|^{-1}{ }^{t} W_{n}(\omega) Q_{0} W_{n}(\omega)$ converges to a matrix with rank $p$, and so the rank of any limit point of $\left\{\left(\left\|W_{n}(\omega)\right\|^{-1} W_{n}(\omega)\right\}_{n=1}^{\infty}{ }_{k}\right.$ is greater than or equal to $p$. This contradicts Theorem 3.1 in [2].

Combining Corollary (2.12) and Theorem (2.14), we have the following.

(2.15) Corollary。 Let $T$ be the semigroup in $G L\left(V_{0}\right)$ generated by $\left\{Y_{1}, \cdots, Y_{N}\right\}$ $\subset G L\left(V_{0}\right)$. Suppose that $\left\{Y_{1}, \cdots, Y_{N}\right\}$ is strongly irreducible and there is a sequence $\left\{M_{n}\right\}_{n=1}^{\infty}$ in $T$ such that $\left\|M_{n}\right\|^{-1} M_{n}$ converges to a matrix whose rank is one. Then $\mu=\nu$, or $\mu$ and $\nu$ are mutually singular.

\section{§3. Stochastic Matrices}

Now let $D, N, M$ be integers with $D \leqq N$ and $D \leqq M$. Let 1 be a vector in $\boldsymbol{R}^{M}$ given by $\mathbb{1}={ }^{t}(1, \cdots, 1)$. Let $V_{0}=\left\{x \in \boldsymbol{R}^{M} ; \sum_{j=1}^{M} x_{j}=0\right\}$ and $P$ be the orthogonal projection in $\boldsymbol{R}^{M}$ onto $V_{0}$. Let $w_{k}, k=1, \cdots, N$, be positive numbers with $\sum_{k=1}^{N} w_{k}=1$. Finally, let $A_{k}, k=1, \cdots, N$ be $M \times M$ matrices satisfying the following conditions.

(A-1) Let $A_{k}=\left(a_{\imath \jmath}^{(k)}\right), i, j=1, \cdots, M$ and $k=1, \cdots, N$. Then

(1) $a_{i j}^{(k)} \geqq 0$ and $\sum_{j=1}^{M} a_{i j}^{(k)}=1$,

(2) $a_{k k}^{(k)}=1$ for $k=1, \cdots, D$,

(3) for any $j=1, \cdots, M$, there is a pair $(i, k) \in\{1, \cdots, D\} \times\{1, \cdots, N\}$ such that $a_{i j}^{(k)}=1$.

(A-2) There are $C \in(0, \infty)$ and $\gamma \in(0,1)$ such that

$$
\left\|\left(P A_{i_{1}} P\right) \cdots\left(P A_{i_{n}} P\right)\right\|_{V_{0} \rightarrow V_{0}} \leqq C \cdot \gamma^{n}
$$

for any $n \geqq 1$ and $i_{1}, \cdots, 1_{n}=1, \cdots, N$.

(A-3) $P A_{2} P, i=1, \cdots, N$, is invertible in $V_{0}$ and $\left\{P A_{1} P, \cdots, P A_{N} P\right\}$ is irreducible in $V_{0}$.

Then we can apply the results in Section 1 , letting $Y_{k}=P A_{k} P, k=1, \cdots, N$. We use the same notation as in Section 1.

We also assume the following.

(A-4) The $M \times M$ matrix $-P Q_{0} P$ is Markov generator, i.e., if we let $q_{i j}=$ $\left(P Q_{0} P\right)_{i j}, i, j=1, \cdots, M$, then $q_{i j} \leqq 0, i \neq j$.

Then we have the following.

(3.1) Theorem. There is a continuous function $f: \Omega \times \boldsymbol{R}^{M} \rightarrow \boldsymbol{R}$ such that $f\left(\omega^{\prime}, x\right) \mathbb{1}$ $=\lim _{n \rightarrow \infty} A_{\omega_{n}^{\prime}} A_{\omega_{n-1}^{\prime}} \cdots A_{\omega_{1}^{\prime}} x$ for any $\omega^{\prime} \in \Omega$ and $x \in \boldsymbol{R}^{M}$. 
Proof. Note that $A_{k} \mathbf{1}=\mathbf{1}, P A_{k}=P A_{k} P, k=1, \cdots, N$. Let $|x|_{\infty}=\max \left\{\left|x_{i}\right|\right.$; $i=1, \cdots, M\}$ for $x \in \boldsymbol{R}^{M I}$. Then $|P x|_{\infty} \leqq|x|_{\infty}$ and $\left|A_{k} x\right|_{\infty} \leqq|x|_{\infty}, k=1, \cdots, N$ and $x \in \boldsymbol{R}^{M}$. Also, there is a $\delta>0$ such that $\delta\|x\| \leqq|x|_{\infty} \leqq \delta^{-1}\|x\|$ for any $x \in \boldsymbol{R}^{M}$.

Let $\tilde{f}_{n}\left(\omega^{\prime}, x\right)=A_{\omega_{n}^{\prime}} A_{\omega_{n-1}^{\prime}} \cdots A_{\omega_{1}^{\prime}} x$ for any $n \geqq 1, \omega^{\prime} \in \Omega$ and $x \in \mathbb{R}^{M I}$. Then we have

$$
\left|P \tilde{f}_{n}\left(\omega^{\prime}, x\right)\right|_{\infty} \leqq \delta^{-1}\left\|\left(P A_{\omega_{n}^{\prime}} P\right) \cdots\left(P A_{\omega_{1}^{\prime}} P\right) x\right\| \leqq \delta^{-2} C \cdot \gamma^{n}|x|_{\infty}
$$

for $n \geqq 1$, and

$$
\begin{aligned}
& \left|\tilde{f}_{m}\left(\omega^{\prime}, x\right)-\tilde{f}_{n}\left(\omega^{\prime}, x\right)\right|_{\infty} \\
\leqq & \left|\left(A_{\omega_{m}^{\prime}} \cdots A_{\omega_{n+1}^{\prime}}-I\right)(I-P) \tilde{f}_{n}\left(\omega^{\prime}, x\right)\right|_{\infty}+\left|A_{\omega_{m}^{\prime}} \cdots A_{\omega_{n+1}^{\prime}} P \tilde{f}_{n}\left(\omega^{\prime}, x\right)\right|_{\infty} \\
\leqq & 2 \delta^{-2} C \cdot \gamma^{n}|x|_{\infty}
\end{aligned}
$$

for $m \geqq n$. These imply our assertion.

From the assumption (I), there is a pair $(i(j), k(j)) \in\{1, \cdots, D\} \times\{1, \cdots, M\}$ such that $a_{i(j)\}}^{(k(j))}=1$ for each $j=1, \cdots, M$. We define $\sigma:\{1, \cdots, M\} \rightarrow \Omega$ by $\sigma(j)=(k(j), i(j), i(j), i(j), \cdots), j=1,2, \cdots, M$.

Then we have

$$
f(\sigma(j), x)=x_{\jmath}, \quad j=1, \cdots, M \text { and } x \in \boldsymbol{R}^{M} .
$$

For $\omega, \omega^{\prime} \in \Omega$ and $n \geqq 0$, we define $\left[\omega, \omega^{\prime}\right]_{n} \in \Omega$ by

Then we see that

$$
\left(\left[\omega, \omega^{\prime}\right]_{n}\right)_{m}= \begin{cases}\omega_{m} & \text { if } m \leqq n \\ \omega_{m-n}^{\prime} & \text { if } m>n .\end{cases}
$$

$$
f\left([\omega, \sigma(j)]_{n}, x\right)=\left(A_{\omega_{n}} \cdots A_{\omega_{1}} x\right)_{j}
$$

for any $n \geqq 1, \omega \in \Omega$ and $j=1, \cdots, M$.

This implies that

$$
P x=\left(P A_{\omega_{1}} P\right)^{-1} \cdots\left(P A_{\omega_{n}} P\right)^{-1} P \cdot{ }^{t}\left(f\left([\omega, \sigma(1)]_{n}, x\right), \cdots, f\left([\omega, \sigma(M)]_{n}, x\right)\right)
$$

for any $n \geqq 0, \omega \in \Omega$ and $x \in \boldsymbol{R}^{M}$.

Let $\mathscr{D}_{0}^{(n)}, n \geqq 1$, be the set of functions $g$ in $\Omega$ such that there is an $\mathscr{F}_{1}^{n}$-measurable map $x: \Omega \rightarrow \boldsymbol{R}^{M}$ such that $g(\omega)=f\left(T^{n} \omega ; x(\omega)\right), \omega \in \Omega$. Since $f(\omega, x)=f\left(T \omega, A_{\omega_{1}} x\right)$, we see that $\mathscr{D}_{0}^{(n)} \subset \mathscr{D}_{0}^{(n+1)}$. Let $\mathscr{D}_{0}=\bigcup_{n} \mathscr{D}_{0}^{(n)}$. Then $\mathscr{D}_{0}$ is a vector space.

(3.5) Lemma. (1) Let $x: \Omega \rightarrow \boldsymbol{R}^{M}$ be $\mathscr{I}_{1}^{n}$-measurable function $\boldsymbol{R}^{M}$ and let $g(\boldsymbol{\omega})=$ $f\left(T^{n} \omega, x(\omega)\right), \omega \in \Omega$. Then

$$
P x(\omega)=\left(P A_{\omega_{n+1}} P\right)^{-1} \cdots\left(P A_{\omega_{m}} P\right)^{-1} P \cdot{ }^{t}\left(g\left([\omega, \sigma(1)]_{m}\right), \cdots, g\left([\omega, \sigma(M)]_{m}\right)\right)
$$

for any $m \geqq n$. In particular, for any $g \in \mathscr{D}_{0}$ and $\omega \in \Omega$ 


$$
X(\omega ; g)=\lim _{m \rightarrow \infty}\left(P A_{\omega_{1}} P\right)^{-1} \cdots\left(P A_{\omega_{m}} P\right)^{-1} P \cdot t\left(g\left([\omega, \sigma(1)]_{m}\right), \cdots, g\left([\omega, \sigma(M)]_{m}\right)\right)
$$

exists.

(2) We define a signed measure $\mu^{\left[g_{1}, g_{2}\right]}$ in $\Omega$ for any $g_{1}, g_{2} \in \mathscr{D}$ by

$$
\mu^{\left[g_{1}, g_{2}\right]}(d \omega)=\left(X\left(\omega ; g_{1}\right), Z(\omega) X\left(\omega ; g_{2}\right)\right) \mu(d \omega) .
$$

Then we have

$$
\begin{aligned}
& \int_{\Omega} \varphi(\omega) \mu^{\left[g_{1}, g_{2}\right]}(d \omega) \\
= & \lim _{n \rightarrow \infty} \lambda^{-n} \int_{\Omega} \nu(d \omega) \varphi(\omega) \sum_{\alpha, \beta=1}^{M}\left(P Q_{0} P\right)_{a \beta} g_{1}\left([\omega, \sigma(\alpha)]_{n}\right) g_{2}\left([\omega, \sigma(\beta)]_{n}\right)
\end{aligned}
$$

for any continuous function $\varphi$ in $\Omega$.

Proof. The assertion (1) is obvious from (3.3). Let $x_{i}: \Omega \rightarrow \mathbb{R}^{M}, i=1,2$, be $\mathscr{F}_{1}^{n}$-measurable and let $g_{i}(\omega)=f\left(T^{n} \omega, x_{i}(\omega)\right)$. Then we see that

$$
\begin{aligned}
& \int_{\Omega} \varphi(\omega) \mu^{\left[g_{1}, g_{2}\right]}(d \omega) \\
= & \lim _{m \rightarrow \infty} \int_{\Omega} \varphi(\omega)\left(X\left(\omega ; g_{1}\right), Z_{n+m}(\omega) X\left(\omega ; g_{2}\right)\right) \mu(d \omega) \\
= & \lim _{m \rightarrow \infty} \int_{\Omega} \varphi(\omega)\left(X\left(\omega ; g_{1}\right),{ }^{t} W_{n+m}(\omega) Q_{0} W_{n+m}(\omega) X\left(\omega ; g_{2}\right)\right) \lambda^{-(n+m)} \nu(d \omega) \\
= & \lim _{m \rightarrow \infty} \lambda^{-(n+m)} \int_{\Omega} \varphi(\omega)\left(P^{t}\left(g\left([\omega, \sigma(1)]_{n+m}\right), \cdots, g\left([\omega, \sigma(M)]_{n+m}\right)\right),\right. \\
\left.\qquad Q_{0} P^{t}\left(g\left([\omega, \sigma(1)]_{n+m}\right), \cdots, g\left([\omega, \sigma(M)]_{n+m}\right)\right)\right) \nu(d \omega) & \lim _{m \rightarrow \infty} \lambda^{-m} \int_{\Omega} \nu(d \omega) \varphi(\omega) \sum_{\alpha, \beta=1}^{M}\left(P Q_{0} P\right)_{\alpha \beta} g_{1}\left([\omega, \sigma(\alpha)]_{m}\right) g_{2}\left([\omega, \sigma(\beta)]_{m}\right)
\end{aligned}
$$

for any continuous function $\varphi$ in $\Omega$. This implies our assertion (2).

We define a map $\tilde{\mathcal{E}}: \mathscr{D}_{0} \times \mathscr{D}_{0} \rightarrow \boldsymbol{R}$ by $\tilde{\mathcal{E}}(g, h)=\int_{\Omega} d \mu^{[g, h]}$. The following is obvious from Lemma (3.5).

(3.9) Proposition. (1) $\tilde{\mathcal{E}}: \mathscr{D}_{0} \times \mathscr{D}_{0} \rightarrow \boldsymbol{R}$ is a symmetric bilinear form.

(2) $\tilde{\varepsilon}(g, g) \geqq 0, g \in \mathscr{D}_{0}$.

(3) If $g, h \in \mathscr{D}_{0}$ and the support of $g$ and $h$ are disjoint, then $\tilde{\varepsilon}(g, h)=0$.

\section{$\S 4$. Restriction of Space and Dirichlet Forms}

The bilinear form introduced in the previous section is not necessarily closable or Markov. The reason is that $\Omega$ is too discrete in the first place and that the assumption to the matrices $A^{(k)}$ 's too general. To avoid these points, we first connect the points in $\Omega$.

Let $J$ be a family of subsets in $\{1, \cdots, N\} \times\{1, \cdots, D\}$ satisfying the following. 
$(\mathrm{J}-1) \cup J=\{1, \cdots, N\} \times\{1, \cdots, D\} \backslash\{(k, k) ; k=1, \cdots, D\}$ and $\#(J) \geqq M-D$.

(J-2) If $B \in J$, then $\#(B) \geqq 2$.

$(\mathrm{J}-3)$ If $B_{1}, B_{2} \in J$ and $B_{1} \neq B_{2}$, then $B_{1} \cap B_{2}=\varnothing$.

$(\mathrm{J}-4)$ If $B \in J,(k, i),\left(k^{\prime}, i^{\prime}\right) \in B$ and $(k, i) \neq\left(k^{\prime}, i^{\prime}\right)$, then $k \neq k^{\prime}$.

(J-5) (Connectivity Condition) For any $k, k^{\prime} \in\{1, \cdots, N\}$, there are $\left(k_{l}, i_{l}\right) \in$ $\{1, \cdots, N\} \times\{1, \cdots, D\}, l=1, \cdots, 2 n$, such that $k_{1}=k, k_{2 n}=k^{\prime}, k_{2 l}=k_{2 l+1}, l=$ $1, \cdots, n-1$, and $\left(k_{2 l-1}, i_{2 l-1}\right),\left(k_{2 l}, i_{2 l}\right) \in B$ for some $B \in J, l=1, \cdots, n$.

We also assume that an injective map $\gamma:\{D+1, \cdots, M\} \rightarrow J$ is given. Let $\tilde{J}=J \cup\{\{(k, k)\} ; k=1, \cdots, D\}$ and $\tilde{\gamma}:\{1, \cdots, M\} \rightarrow \tilde{J}$ be given by $\tilde{\gamma}(i)=\{(i, i)\}$ if $1 \leqq i \leqq D$, and $\tilde{\gamma}(i)=\gamma(i)$ if $D+1 \leqq i \leqq M$. Finally we assume the following.

$(\mathrm{H}-1) \quad a_{i j}^{(k)}=a_{i^{\prime} j}^{\left(k^{\prime}\right)}, j=1, \cdots, M$, if $(k, i),\left(k^{\prime}, i^{\prime}\right) \in B$ for some $B \in J$.

(H-2) $\quad a_{i j}^{(k)}=1$ if $(k, i) \in \tilde{\gamma}(j), j=1, \cdots, M$.

$(\mathrm{H}-3)$ (harmonicity condition)

$$
\sum_{k=1}^{N} w_{k}\left(P A_{k} x, Q_{0} P y^{(k)}\right)_{V_{0}}=0 \text { for any } x \in \boldsymbol{R}^{M},
$$

provided that $y^{(k)}={ }^{t}\left(y_{1}^{(k)}, \cdots, y_{M}^{(k)}\right) \in \boldsymbol{R}^{M}, k=1, \cdots, N$, satisfies that $y_{i}^{(k)}=0$ if $(k, i) \in \tilde{\gamma}(j)$ for some $j=1, \cdots, M^{\prime}$ and that $y_{i}^{(k)}=y_{i^{\prime}}^{\left(k^{\prime}\right)}$ if $(k, i),\left(k^{\prime}, i^{\prime}\right) \in B$ for some $B \in J$.

(4.1) Remark. (1) (H-2) implies (A-1) (3).

(2) Given $D, N, M$ with $2 \leqq D \leqq N$ and $D \leqq M$, the set $J$ satisfying the conditions $(\mathrm{J}-1)-(\mathrm{J}-5)$, and the injection $\gamma:\{D+1, \cdots, M\} \rightarrow J$, we have algebraic equations (H-1)-(H-3), (A-1)(1), (2), and the following (4.2) for $A_{i}, i=1, \cdots, N, Q_{0}$ and $\lambda$.

$$
\sum_{i=1}^{N} w_{i}{ }^{t}\left(P A_{i} P\right) Q_{0}\left(P A_{i} P\right)=\lambda Q_{0} \text {. }
$$

The existence of suitable $A_{i}$ 's, $Q_{0}$ and $\lambda$ is not obvious. This problem has been essentially solved by Lindstrom [10] for nested fractals (see Section 6). This problem is also discussed essentially in Hattori-Hattori-Watanabe [7] for more general fractals.

Let us introduce an equivalence relation $\sim$ in $\Omega$ by the following: (4.3) $\omega \sim \omega^{\prime}$, if $\omega=\omega^{\prime}$ or if there are an $n \geqq 0$ and $(k, i),\left(k^{\prime}, i^{\prime}\right) \in B$ for some $B \in J$ such that $\omega_{m}=\omega_{m}^{\prime}, 1 \leqq m \leqq n-1, \omega_{n}=k, \omega_{n}^{\prime}=k^{\prime}, \omega_{l}=i$ and $\omega_{l}^{\prime}=i^{\prime}, l \geqq n+1$.

We denote by $S$ the quotient topological space $\Omega / \sim$. It is easy to see that $S$ is a compact Hausdorff space (See Bourbaki [3]). Let $\pi: \Omega \rightarrow S$ be the natural projection. If we let $R=\left\{\omega \in \Omega ; \#\left(\pi^{-1}(\omega)\right) \geqq 2\right\}$, then $R$ is a countable subset of $\Omega$. Therefore we see that $\nu(R)=\mu(R)=0$. So we will sometimes identify $S$ with $\Omega$ when we think of functions defined only $\nu$-a.e. or $\mu$-a.e. Let $\mathscr{D}=$ $\left\{g \in \mathscr{D}_{0} ; g(\boldsymbol{\omega})=g\left(\boldsymbol{\omega}^{\prime}\right)\right.$ if $\left.\boldsymbol{\omega} \sim \boldsymbol{\omega}^{\prime}\right\}$. Then it is easy to see that $f(\cdot, x) \in \mathscr{D}$ for any $x \in \boldsymbol{R}^{M}$. Each element of $\mathscr{D}$ may be regarded as a continuous function in $S$. 
For each $j=1, \cdots, M$, fix an element $(k(j), i(j)) \in \tilde{\gamma}(j)$ and let $\sigma(j)=$ $(k(j), i(j), i(j), \cdots) \in \Omega$. Then $\pi(\sigma(j))$ is independent of the choice of $(k(j), i(j))$ $\in \tilde{\gamma}(j)$. Moreover, $\pi\left([\omega, \sigma(j)]_{n}\right)$ depends only on $\omega, n$ and $j$, and independent of the choice of $(k(j), i(j))$.

For each $g \in C(S)$ and $n \geqq 0$, let $x_{n}(\omega ; g)={ }^{t}\left(g \circ \pi\left([\omega, \sigma(1)]_{n}, \cdots, g \circ \pi\left([\omega, \sigma(M)]_{n}\right)\right)\right.$, and let $S_{n} g(\omega)=f\left(T^{n} \omega ; x_{n}(\omega ; g)\right), \omega \in \Omega$.

(4.4) Lemma. $S_{n} g \in \mathscr{D}$ for any $g \in C(S)$.

Proof. It is obvious that $x_{n}(\cdot ; g): \Omega \rightarrow \mathbb{R}^{11}$ is $\mathscr{F}_{1}^{n}$-measurable. So $S_{n} g \in \mathscr{D}_{0}^{(n)}$. Therefore it is sufficient to show that $S_{n} g(\omega)=S_{n} g\left(\omega^{\prime}\right)$, if $\omega=\omega^{\prime}$. There are two cases.

Case 1. $\omega_{k}=\omega_{k}^{\prime}$ for $k=1, \cdots, n$.

In this case, $S_{n} g(\omega)=S_{n} g\left(\omega^{\prime}\right)$ is obvious.

Case 2. $\omega_{k} \neq \omega_{k}^{\prime}$ for some $k=1, \cdots, n$.

In this case, there are an $m<n$ and $(k, i),\left(k, i^{\prime}\right) \in B$ for some $B$ such that $\omega_{k}=\omega_{k}^{\prime}, k=1, \cdots, m, \omega_{m+1}=k, \omega_{m+1}^{\prime}=k^{\prime}, \omega_{l}=i$ and $\omega_{l}^{\prime}=i^{\prime}, l \geqq m+2$. Then $T^{n} \omega$ $=(i)$ and so $S_{n} g(\omega)=g\left([\omega, \sigma(i)]_{n}\right)=g\left([\omega,(i)]_{n}\right)=g(\omega)$. Similarly $S_{n} g\left(\omega^{\prime}\right)=g\left(\omega^{\prime}\right)$. Therefore $S_{n} g(\omega)=S_{n} g\left(\omega^{\prime}\right)$.

This completes the proof.

(4.5) Lemma. (1) $\tilde{\mathcal{E}}\left(S_{n} g, S_{n} g\right)=\lambda^{-n} \int_{\Omega} \nu(d \omega)\left(P x_{n}(\omega ; g), Q_{0} P x_{n}(\omega ; g)\right)$ for any $g \in$ $C(S)$ and $n \geqq 0$.

(2) $\tilde{\mathcal{E}}\left(S_{n+1} g-S_{n} g, S_{n} g\right)=0, g \in C(S)$ and $n \geqq 0$.

(3) $\tilde{\mathcal{E}}(g, g)=\tilde{\mathcal{E}}\left(S_{1} g, S_{1} g\right)+\sum_{n=1}^{\infty} \tilde{\mathcal{E}}\left(S_{n+1} g-S_{n} g, S_{n+1} g-S_{n} g\right), g \in \mathscr{D}$.

Proof. The assertion (1) is obvious from (3.3) and Lemma (3.5). Note that $S_{n} g(\omega)=f\left(T^{n+1} \omega ; A_{\omega_{n+1}} x_{n}(\omega ; g)\right)$. Therefore we have

$$
\begin{aligned}
& \tilde{\mathcal{E}}\left(S_{n+1} g-S_{n} g, S_{n} g\right) \\
= & \lambda^{-(n+1)} \int_{\Omega} \nu(d \omega)\left(P\left(A_{\omega_{n+1}} x_{n}(\omega ; g)-x_{n+1}(\omega ; g)\right), Q_{0} P A_{\omega_{n+1}} x_{n}(\omega ; g)\right) \\
= & \lambda^{-(n+1)} \int_{\Omega} \nu(d \omega) \sum_{k=1}^{N} w_{k}\left(P\left(A_{k} x_{n}(\omega ; g)-x_{n+1}\left([\omega,(k)]_{n} ; g\right)\right), Q_{0} P A_{k} x_{n}(\omega ; g)\right) .
\end{aligned}
$$

Here $(k)=(k, k, \cdots) \in \Omega$. Let $y^{(k)}=x_{n+1}\left([\omega,(k)]_{n} ; g\right)-A^{(k)} x_{n}(\omega ; g)$. If $(k, i)$ $\in \tilde{\gamma}(j)$, then

$$
y_{i}^{(k)}=g\left(\left[[\omega,(k)]_{n},(i)\right]_{n+1}\right)-g\left([\omega, \sigma(j)]_{n}\right)=0 .
$$

Also, if $(k, i),\left(k^{\prime}, i^{\prime}\right) \in B$ for some $B \in J$, then

$$
y_{i}^{(k)}-y_{i^{\prime}}^{\left(k^{\prime}\right)}=g\left(\left[[\omega,(k)]_{n},(i)\right]_{n+1}\right)-g\left(\left[\left[\omega,\left(k^{\prime}\right)\right]_{n},\left(i^{\prime}\right)\right]_{n+1}\right)=0 .
$$

Therefore by the harmonicity condition $(\mathrm{H}-3)$, we have 


$$
\sum_{k=1}^{N} w_{k}\left(P y^{(h)}, Q_{0} P A_{k} x_{n}(\omega ; g)\right)=0 .
$$

Thus we obtain our assertion (2).

By Lemma (3.5), we have

$$
\begin{aligned}
\tilde{\mathcal{E}}(g, g) & =\lim _{n \rightarrow \infty} \lambda^{-n} \int_{\Omega} \nu(d \omega) \sum_{a, \beta=1}^{M}\left(P Q_{0} P\right)_{\alpha \beta} g\left([\omega, \sigma(\alpha)]_{n}\right) g\left([\omega, \sigma(\beta)]_{n}\right) \\
& =\lim _{n \rightarrow \infty} \tilde{\mathcal{E}}\left(S_{n} g, S_{n} g\right) .
\end{aligned}
$$

Since the assertion (2) implies that

$$
\tilde{\mathcal{E}}\left(S_{n+1} g, S_{n+1} g\right)=\tilde{\mathcal{E}}\left(S_{n} g, S_{n} g\right)+\tilde{\mathcal{E}}\left(S_{n+1} g-S_{n} g, S_{n+1} g-S_{n} g\right),
$$

we have the assertion (3).

Let $\tilde{\nu}$ denotes the probability measure $\nu \circ \pi^{-1}$ in $S$. Then we have the following.

(4.6) Theorem. (1) $\mathscr{D}$ is dense in $C(S)$.

(2) $\left.\tilde{\mathcal{E}}\right|_{\mathscr{D} \times \mathscr{D}}$ is closable in $L^{2}(S ; d \tilde{\nu})$.

(3) Let $\mathcal{E}$ be the smallest closed extension of $\left.\tilde{\mathcal{E}}\right|_{\mathscr{D} \times \mathscr{D}}$. If $g \in C(S)$ and $\sup _{n} \tilde{\mathcal{E}}\left(S_{n} g, S_{n} g\right)<\infty$, then $g \in \mathscr{D}_{\circ m}(\mathcal{E})$ and $\mathcal{E}(g, g)=\lim _{n \rightarrow \infty} \tilde{\mathcal{E}}\left(S_{n} g, S_{n} g\right)$. Moreover, $\mathcal{E}$ is a local regular Dirichlet form in $L^{2}(S ; d \tilde{\nu})$, and satisfies the following.

(4.7) $\quad \mathcal{E}(h, h)=0$ if and only if $h(z)=$ constant for $\tilde{\nu}$-a.e. $z$.

$$
\begin{aligned}
& \int_{S}\left|h(z)-\int_{S} h\left(z^{\prime}\right) \tilde{\mathcal{L}}\left(d z^{\prime}\right)\right|^{2} \tilde{\mathcal{L}}(d z) \leqq C \cdot \mathcal{E}(h, h), \quad h \in \mathscr{D}_{o m}(\mathcal{E}), \\
& \left|h((i))-\int_{S} h(z) \tilde{\mathcal{L}}(d z)\right| \leqq C \cdot \mathcal{E}(h, h)^{1 / 2}, \quad h \in \mathscr{D}_{\circ}(\mathcal{E}) \text { and } i=1, \cdots, D,
\end{aligned}
$$

for some constant $C>0$.

Proof. Let $g \in C(S)$ and $g_{n}$ be as in Lemma (4.4). Then we have $\left\|g \circ \pi-g_{n}\right\|_{C(\Omega)} \rightarrow 0$ as $n \rightarrow \infty$, since $\Omega$ is compact metric space and so $g$ is uniformly continuous. This proves our assertion (1).

By Lemma (4.5) and the fact that $Q_{0}$ is strictly positive, we see that

$$
\sup _{m} \lambda^{-m} \int_{\Omega} \nu(d \omega) \sup _{1 \leqq i, j \leqq D}\left|g\left([\omega,(i)]_{m}\right)-g\left([\omega,(j)]_{m}\right)\right|^{2} \leqq C \cdot \tilde{\varepsilon}(g, g), \quad g \in \mathscr{D},
$$

for some $C<\infty$, and so

$$
\begin{aligned}
& \sup _{m} \lambda^{-m} \int_{\Omega} \nu(d \omega) \sum_{k=1}^{N} \sup _{1 \leqq 2, j \leqq D}\left|g\left([\omega,\langle k, i\rangle]_{m}\right)-g\left([\omega,\langle k, j\rangle]_{m}\right)\right|^{2} \\
& \leqq C \cdot \tilde{\mathcal{E}}(g, g), \quad g \in \mathscr{D},
\end{aligned}
$$

for some $C<\infty$. Here $\langle k, i\rangle=(k, i, i, i, \cdots) \in \Omega$. 
Note that $g\left([\omega,\langle k, i\rangle]_{m}\right)=g\left(\left[\omega,\left\langle k^{\prime}, i^{\prime}\right\rangle\right]_{m}\right)$ if $(k, i),\left(k^{\prime}, i^{\prime}\right) \in B$ for some $B \in J$. Therefore using the assumption $(\mathrm{J}-5)$, we have

$$
\begin{aligned}
& \sup _{m} \lambda^{-m} \int_{\Omega} \nu \circ \pi^{-1}(d \omega) \sup _{1 \leqq k \leqq N} \sup _{1 \leqq i, j \leqq D}\left|g\left([\omega,\langle k, i\rangle]_{m}\right)-g\left([\omega,(j)]_{m}\right)\right|^{2} \\
& \leqq C \cdot \tilde{\mathcal{E}}(g, g), \quad g \in \mathscr{D},
\end{aligned}
$$

for some $C<\infty$, and so

$$
\int_{\Omega} \nu(d \omega) \mid g\left([\omega,(i)]_{m+1}\right)-g\left([\omega,(i)]_{m}\right)_{1}^{\prime 2} \leqq C \lambda^{m} \cdot \tilde{\mathcal{E}}(g, g), \quad g \in \mathscr{D}
$$

for all $m \geqq 0$ and $i=1, \cdots, D$.

By the assumption (A-2) in Section 3, we see that

$$
\lambda^{n} \cdot \operatorname{trace} Q_{0}=\sum_{i_{1}, \cdots, i_{n}=1}^{M} w_{i_{1}} \cdots w_{i_{n}} \operatorname{trace}\left({ }^{t}\left(A_{i_{1}} \cdots A_{i_{n}}\right) Q_{0}\left(A_{i_{1}} \cdots A_{i_{n}}\right)\right) \leqq C \cdot \gamma^{2 n}
$$

for all $n \geqq 1$. This shows that $\lambda \leqq \gamma^{2}<1$. Therefore we have

$$
\int_{\Omega} \nu(d \omega)\left|g(\omega)-g\left([\omega,(i)]_{m}\right)\right|^{2} \leqq C(1-\lambda)^{-1} \lambda^{m} \cdot \tilde{\mathcal{E}}(g, g), \quad g \in \mathcal{2} .
$$

Thus by (4.10), we see that there is a $C<\infty$ such that

$$
\int_{\Omega} \nu(d \omega)\left|g(\omega)-g\left([\omega, \sigma(j)]_{m}\right)\right|^{2} \leqq C \lambda^{m} \cdot \tilde{\varepsilon}(g, g)
$$

for any $g \in \mathscr{D}, m \geqq 0$ and $j=1, \cdots, M$.

Now suppose that $g_{n} \in \mathscr{D}, n \geqq 1,\left\|g_{n}\right\|_{L^{2}(d \tilde{\nu})} \rightarrow 0$ as $n \rightarrow \infty$, and $\tilde{\varepsilon}\left(g_{n}-g_{m}, g_{n}-g_{m}\right)$ $\rightarrow 0$ as $n, m \rightarrow \infty$. Then by (4.12) we see that $S_{m} g_{n}(\omega) \rightarrow 0$ as $n \rightarrow \infty$ for any $\omega \in \Omega$ and $j=1, \cdots, M$. By Lemma (4.5), we have

$$
\begin{aligned}
\varlimsup_{n, m \rightarrow \infty} \sum_{k=1}^{\infty} \mid \tilde{\mathcal{E}}\left(\left(S_{k+1}-S_{k}\right) g_{n},\left(S_{k+1}-S_{k}\right) g_{n}\right)^{1 / 2} \\
-\left.\tilde{\mathcal{E}}\left(\left(S_{k+1}-S_{k}\right) g_{m},\left(S_{k+1}-S_{k}\right) g_{m}\right)^{1 / 2}\right|^{2}=0 .
\end{aligned}
$$

Therefore, we see that

$$
\begin{aligned}
\lim _{n \rightarrow \infty} \tilde{\mathcal{E}}\left(g_{n}, g_{n}\right) & =\lim _{k \rightarrow \infty} \lim _{n \rightarrow \infty}\left(\tilde{\mathcal{E}}\left(S_{1} g_{n}, S_{1} g_{n}\right)+\sum_{l=1}^{k} \tilde{\mathcal{E}}\left(\left(S_{l+1}-S_{l}\right) g_{n},\left(S_{l+1}-S_{l}\right) g_{n}\right)\right) \\
& =0 .
\end{aligned}
$$

This proves our assertion (2).

Suppose that $g \in C(S)$ and $\sup _{n} \tilde{\mathcal{E}}\left(S_{n} g, S_{n} g\right)<\infty$. Since $S_{n} g(\omega) \rightarrow g(\omega)$ uniformly, we see that $S_{n} g \rightarrow g$ in $L^{2}(S ; d \tilde{\nu})$. By Lemma (4.5), we see that $\tilde{\mathcal{E}}\left(S_{n} g-S_{m} g\right.$, $\left.S_{n} g-S_{m} g\right) \rightarrow 0$ as $n, m \rightarrow \infty$. Therefore we see that $g \in \mathscr{D}_{o m}(\mathcal{E})$ and $\mathcal{E}(g, g)$ $=\lim _{n \rightarrow \infty} \tilde{\varepsilon}\left(S_{n} g, S_{n} g\right)$.

Now let $g \in \mathscr{D}$ and $\varphi \in C^{1}(\boldsymbol{R})$ with $\varphi(0)=0$ and $\left|\varphi^{\prime}(t)\right| \leqq 1, t \in \boldsymbol{R}$. Then we have by the assumption (A-4) 


$$
\begin{aligned}
& \mathcal{E}\left(S_{n}(\varphi \circ g), S_{n}(\varphi \circ g)\right) \\
= & \lambda^{-n} \int_{\Omega} \nu(d \omega) \sum_{\alpha, \beta=1}^{M}\left(P Q_{0} P\right)_{a \beta} \cdot \varphi\left(g\left([\omega ; \sigma(\alpha)]_{n}\right)\right) \varphi\left(g\left([\omega ; \sigma(\beta)]_{n}\right)\right) \\
\leqq & \lambda^{-n} \int_{\Omega} \nu(d \omega) \sum_{\alpha, \beta=1}^{M}\left(P Q_{0} P\right)_{\alpha \beta} \cdot g\left([\omega ; \sigma(\alpha)]_{n}\right) g\left([\omega ; \sigma(\beta)]_{n}\right)=\mathcal{E}\left(S_{n} g, S_{n} g\right) .
\end{aligned}
$$

Thus we see that $\varphi \circ g \in \mathscr{D}_{\circ m}(\mathcal{E})$ and $\mathcal{E}(\varphi \circ g, \varphi \circ g) \leqq \mathcal{E}(g, g)$. This implies that $\mathcal{E}$ is Markov.

Then by Proposition (3.9) and Fukushima [5] Theorem 2.1.1, we see that the smallest closed extension $\mathcal{E}$ is a local Dirichlet form.

Also (4.11) leads to (4.9), and by them we have

$$
\int_{\Omega} \nu(d \omega)\left|g(\omega)-\int_{\Omega} g\left(\omega^{\prime}\right) \nu\left(d \omega^{\prime}\right)\right|^{2} \leqq 4 C \cdot \tilde{\varepsilon}(g, g), \quad g \in \mathscr{D} .
$$

This implies (4.8). (4.7) follows from (4.8) immediately.

This completes the proof.

(4.13) Lemma. (1) If $\omega_{1} \sim \omega_{2}$ in $\Omega$, then $\left[\omega, \omega_{1}\right]_{n} \sim\left[\omega, \omega_{2}\right]_{n}$ for any $n \geqq 1$ and $\omega \in \Omega$.

(2) If $g \in \mathscr{D}_{\circ m}(\mathcal{E})$, then $g\left([\omega, \cdot]_{n}\right) \in \mathscr{D}_{o m}(\mathcal{E})$ for any $n \geqq 1$ and $\omega \in \Omega$, and

$$
\int_{\Omega} \mathcal{E}\left(g\left([\omega, \cdot]_{n}, g\left([\omega, \cdot]_{n}\right)\right) \nu(d \omega)=\lambda^{n} \mathcal{E}(g, g) .\right.
$$

Here we identify functions on $S$ with ones on $\Omega$.

Proof. (1) is obvious. Also it is easy to see that $g\left([\omega, \cdot]_{n}\right) \in \mathscr{D}$ for any $g \in \mathscr{D}$. Then (2) follows from Lemma (3.5) and Theorem (4.6).

For each $n \geqq 0$, let $F_{n}=\left\{[\omega,(i)]_{n} \in \Omega ; \omega \in \Omega, i=1, \cdots, D\right\}$. Then $F_{n}$ is a finite subset in $\Omega$. Also, for each $n \geqq 0$ and $\omega \in \Omega$, let $\Omega_{\omega}^{(n)}=\left\{\left[\omega, \omega^{\prime}\right]_{n} \in \Omega ; \omega^{\prime} \Omega\right\}$. Then we have the following.

(4.15) Lemma. (1) $g(\omega)$ is well defined for any $g \in \mathscr{D o m}(\mathcal{E})$ and $\omega \in \bigcup_{n} F_{n}$.

(2) $\pi\left(\Omega_{\omega}^{(n)}\right) \cap \pi\left(\Omega_{\omega^{\prime}}^{(n)}\right) \subset \pi\left(F_{n}\right)$ for any $n \geqq 1$ and $\omega, \omega^{\prime} \in \Omega$ for which $[\omega,(1)]_{n} \neq$ $\left[\omega^{\prime},(1)\right]_{n}$.

Proof. (1) follows from (4.9) and (4.14). (2) follows from the fact that if $\left[\omega, \omega^{\prime \prime}\right]_{m} \sim\left[\omega^{\prime}, \omega^{\prime \prime}\right]_{m}$, then $m \leqq n$.

The following is obvious from Lemma (4.13) and Lemma (4.15).

(4.16) Theorem. Let $\omega \in \Omega$ and $n \geqq 1$. If $g \in \mathscr{D}_{\circ \mathrm{m}}(\mathcal{E})$ satisfies that

(1) supp $g \subset \pi\left(\Omega_{\omega}^{(n)}\right)$ ע-a.e. and

(2) $g\left(\omega^{\prime}\right)=0$ for any $\omega^{\prime} \in F_{n}$,

then

$$
\mathcal{E}\left(g\left([\omega, \cdot]_{n}\right), g\left([\omega, \cdot]_{n}\right)\right)=\lambda^{n}\left(w_{\omega_{1}} \cdots w_{\omega_{n}}\right)^{-1} \mathcal{E}(g, g)
$$




\section{$\S 5$. Associated Dififusion Process}

Since $\mathcal{E}$ is a local regular Dirichlet form in $L^{2}(S ; d \tilde{\mathcal{\nu}})$ there is a diffusion process in $S$ associated to $\varepsilon$. Then we can apply the theory of Dirichlet form, especially the results in Fukushima [5] Chapter 5. We use the notions there.

(5.1) Lemma. There is a measurable map $Y(\cdot ; g): \Omega \rightarrow V_{0} \subset \mathbb{R}^{M}$ for each $g \in \mathscr{D o m}_{\mathrm{om}}(\mathcal{E})$ satisfying the following.

(1) $\mu^{\left[g_{1}, g_{2}\right]}(d \omega)=\left(Y\left(\omega ; g_{1}\right), Z(\omega) Y\left(\omega ; g_{2}\right)\right) \mu(d \omega), g_{1}, g_{2} \in D_{o m}(\mathcal{E})$.

(2) $Y\left(\omega ; g_{1}+g_{2}\right)=Y\left(\omega ; g_{1}\right)+Y\left(\omega ; g_{2}\right) \mu$-a.e. $\omega, g_{1}, g_{2} \in \mathscr{D}_{\circ}(\mathcal{E})$.

(3) $Y(\omega ; g) \in$ Image $Z(\omega) \mu$-a.e. $z, g \in \mathscr{D}_{o m}(\mathcal{E})$.

(4) $Y(\omega ; f(\cdot ; x))=P(\omega) x \mu$-a.e. $\omega$ for any $x \in V_{0}$. Here $P(\omega)$ is the orthogonal projection in $V_{0}$ onto Image $Z(\omega)$.

Proof. Since $Z(\omega)$ is a non-negative symmetric matrix, $P(\omega)=$ $\lim _{\varepsilon \downarrow 0}(\varepsilon I+Z(\omega))^{-1} Z(\omega) \mu$-a. e. $z$. Let $\lambda(\omega)$ be the minimal eigen value of $Z(\omega)+$ $(I-P(\omega))$. Then $\lambda(\omega)>0$. For any $g \in \mathscr{D}$, let $Y(\omega ; g)=P(\omega) X(\omega ; g)$, where $X(\omega ; g)$ is one given in Lemma (3.5). Then we have

$$
\begin{aligned}
\mathcal{E}(g, g) & =\int_{\Omega}(Y(\omega ; g), Z(\omega) Y(\omega ; g)) \mu(d \omega) \\
& \geqq \int_{\Omega}|Y(\omega ; g)|^{2} \lambda(\omega) \mu(d \omega) .
\end{aligned}
$$

Note that $X(\omega ; g), g \in \mathscr{D}$, is linear in $g$. So defining $Y(\omega ; g), g \in \mathscr{D}_{\circ}(\mathcal{E})$, by the limit in $L^{2}(\Omega ; \lambda(\omega) d \mu)$, we obtain our assertion.

(5.3) Lemma. Let $(P, \mathscr{B}, \Omega)$ be a probability measure, $n$ and $m$ be natural numbers with $m \leqq n-1$, and $A: \Omega \rightarrow \boldsymbol{R}^{n} \otimes \boldsymbol{R}^{n}$ be a measurable map such that $P[\operatorname{rank} A=m, A$ is a nonnegative definite matrix $]=1$. Then there is an $m$ dimensional vector subspace in $\mathbb{R}^{n}$ such that $P[(A(\omega) v, v)>0$ for any $v \in V \backslash\{0\}]=1$.

Proof. We prove this lemma by induction in $k=n-m$.

Step 1. We prove our lemma in the case that $k=1$.

Let $\mathbb{P}^{n-1}$ be the projective space of dimension $n-1$. Then we can identify $\mathbb{P}^{n-1}$ with the set of one dimensional vector subspaces in $\mathbb{R}^{n}$. Let $\Phi(\boldsymbol{\omega})=$ $\left\{v \in \mathbb{R}^{n} ;(A(\omega) v, v)=0\right\}$. Then we may regard $\Phi$ as a measurable map from $\Omega$ into $\mathbb{P}^{n-1}$ except on a null set. Note that if $v$ and $\Phi(\omega)$ are linearly independent, then $(A(\omega) v, v) \neq 0$.

Now we show that for any $l=1, \cdots, n-1$, there is an $l$-dimensional subspace $W_{l}$ in $\mathbb{R}^{n}$ such that $P\left(\Phi^{-1}\left(W_{l}\right)\right)=0$ by induction in $l$. This is obvious in the case where $l=1$. Now suppose that this statement is correct for $l$ with $1 \leqq l \leqq n-2$. Then, let $B_{l}=\left\{v \in \mathbb{P}^{n-1} ;(v, u)=0\right.$ for all $\left.u \in W_{l}\right\}$. Then $B_{l}$ is uncountable. Note that if $v_{1}, v_{2} \in B_{l}$ and $v_{1} \neq v_{2}$, then $\left(W_{l}+\mathbb{R} v_{1}\right) \cap\left(W_{l}+\mathbb{R} v_{2}\right)=W_{l}$. 
Since $P\left(\Phi^{-1}\left(W_{l}\right)\right)=0$, there is a $v \in B_{l}$ such that $P\left(\Phi^{-1}\left(W_{l}+\boldsymbol{R} v\right)\right)=0$. This proves that our statement is correct for $l+1$.

Therefore there is an $m$-dimensional subspace $V$ such that $P\left(\Phi^{-1}(V)\right)=0$, and so $P[(A(\omega) v, v)>0$ for all $v \in V \backslash\{0\}]=1$.

Step 2. Now suppose that $n-m \geqq 2$. Then by using measurable selection theorem, we see that there is a measurable map $X: \Omega \rightarrow \boldsymbol{R}^{n}$ such that $P[\|X(\omega)\|=1$, $(A(\omega) X(\omega), X(\omega))=0]=1$. Let $\tilde{A}(\boldsymbol{\omega})=A(\boldsymbol{\omega})+X(\boldsymbol{\omega}) \otimes X(\boldsymbol{\omega})$. Then from the assumption of the induction, we see that there is an $(m+1)$-dimensional subspace $V^{\prime}$ such that $P\left[(A(\omega) v, v)>0\right.$ for all $\left.v \in V^{\prime}\right]=1$. Let $\bar{A}(\omega)=\left.P_{V^{\prime}} A(\omega)\right|_{V^{\prime}}$. Here $P_{V^{\prime}}$ is an orthogonal projection onto $V^{\prime}$. Then, since $\operatorname{dim} V^{\prime}=m+1$ and $P[\operatorname{rank} \bar{A}(\omega)=m]$ $=1$, applying the result in Step 1 , we see that there is an $m$-dimensional vector subspace $V$ in $V^{\prime}$ such that $P[(\bar{A}(\omega) v, v)>0$ for all $v \in V]=1$.

This completes the proof.

(5.4) Theorem. Let $p$ be the index as in Definition (1.10). Then we have the following.

(1) There are $x_{1}, \cdots, x_{p} \in V_{0} \subset \boldsymbol{R}^{M}$ such that $Z(\omega) x_{1}, \cdots, Z(\omega) x_{p}$ are linearly independent for $\mu$-a.e. $\omega$.

(2) There are $P_{i}$-martingales $\left\{M_{t}^{i}\right\}_{i=1, \ldots, p}$ satisfying the following.

(i) $\left\langle M^{i}, M^{j}\right\rangle_{t}=\delta_{i j}\left\langle M^{1}, M^{1}\right\rangle_{t}, t \geqq 0, P_{\tilde{i}-}$..$s$.

(ii) For any $g \in \mathscr{D o m}(\mathcal{E})$, there is a measurable map $h_{i}(\cdot ; g): S \rightarrow \boldsymbol{R}, i=1, \cdots, p$ such that

$$
M_{t}^{[g]}=\sum_{i=1}^{p} \int_{0}^{t} h_{\imath}\left(X_{s} ; g\right) d M_{s}^{i}, \quad t \geqq 0, P_{\tilde{\nu}-} a . s .
$$

In particular, the martingale dimension of the diffusion process $P_{\mu}$ is the index $p$ (see Davis-Varaiya [4] for the definition of the martingale dimension).

Proof. First note that $\mu$ is a smooth measure in the sense of Fukushima [5]. In fact, if we take $y_{i} \in \boldsymbol{R}^{M}, i=1, \cdots, n$, such that $Q_{1}=\sum_{i=1}^{n} y_{i} \otimes y_{i}$, then $\mu=\sum_{i=1}^{n} \mu^{\left[f\left(\cdot, y_{i}\right)\right]}$.

By Theorem (2.8), we have that $\operatorname{rank} Z(\omega)=p$ for $\mu$-a. e. $\omega$. Therefore from Lemma (5.3) there is a $p$-dimensional subspace $V$ in $V_{0}$ such that $\mu[(v, Z(\omega) v)>0$ for all $v \in V \backslash\{0\}]=1$. Let $\left\{x_{1}, \cdots, x_{p}\right\}$ be a linear basis of $V$. Let $A(\omega)=$ $\left(A_{i j}(\omega)\right)_{i, j=1, \ldots, p}=\left(\left(x_{i}, Z(\omega) x_{j}\right)\right)_{i, j=1, \ldots, p}$. Then $A(\omega)$ is strictly positive symmetric matrix for $\mu$-a.e. $\omega$. Let $B(\omega)=A(\omega)^{-1 / 2} \mu$-a. e. $\omega$, and let

$$
M_{t}^{i}=\sum_{j=1}^{p} \int_{0}^{t} B_{\imath \jmath}\left(X_{s}\right) d M_{s}^{\left[f\left(\cdot ; x_{j}\right)\right], \quad t \geqq 0 .}
$$

Then by Lemma (5.1), for each $i, j=1, \cdots, p$, the associated measure with an additive functional $\left\langle M^{i}, M^{j}\right\rangle_{t}$ is given by 


$$
\sum_{k, l=1}^{p} B_{i k}(\omega) B_{j l}(\omega)\left(x_{k}, Z(\omega) x_{l}\right) \mu(d \omega)=\delta_{i j} \mu(d \omega)
$$

This proves (2)(i).

Note that

$$
Z(\omega) y=\sum_{i, j=1}^{p}\left(y, Z(\omega) x_{\jmath}\right)\left(A(\omega)^{-1}\right)_{i, j} \cdot Z(\omega) x_{i}, \quad y \in V_{0} .
$$

Therefore let $h_{i}(\omega ; g)=\sum_{j=1}^{p} B_{i, j}(\omega)\left(Y(\omega ; g), Z(\omega) x_{j}\right)$ for each $g \in \mathscr{D o m}_{o}(\mathcal{E})$. Then we have

$$
Z(\omega)\left(\sum_{i, k=1}^{p} h_{i}(\omega ; g) B_{i k}(\omega) x_{k}\right)=Z(\omega) Y(\omega ; g) \quad \mu \text {-a. е. } \omega \text {. }
$$

Therefore by the results in Fukushima [5] Chapter 5, we have

$$
\begin{aligned}
& E_{\tilde{\nu}}\left[\left\langle M^{[g]}-\sum_{i=1}^{p} \int_{0}^{\cdot} h_{i}\left(X_{s} ; g\right) d M_{s}^{i}, M^{[g]}-\sum_{i=1}^{p} \int_{0}^{\cdot} h_{\imath}\left(X_{s} ; g\right) d M_{s}^{i}\right\rangle_{1}\right] \\
= & E_{\tilde{\nu}}\left[\left\langle M^{[g]}, M^{[g]}\right\rangle_{1}\right]+\sum_{i, j=1}^{p} E_{\tilde{\nu}}\left[\left\langle\int_{0}^{\cdot} h_{i}\left(X_{s} ; g\right) d M_{s}^{i}, \int_{0}^{\cdot} h_{\jmath}\left(X_{s} ; g\right) d M_{s}^{\jmath}\right\rangle_{1}\right] \\
& \quad-2 \sum_{i=1}^{p} E_{\tilde{\nu}}\left[\left\langle\int_{0}^{\cdot} h_{\imath}\left(X_{s} ; g\right) d M_{s}^{i}, M^{[g]}\right\rangle_{1}\right] \\
= & \int_{\Omega_{0}} d \mu^{[g, g]}+\sum_{i=1}^{p} \int_{\Omega_{0}} h_{i}(\omega ; g) h_{i}(\omega ; g) d \mu \\
= & \quad-2 \sum_{i, j=1}^{p} \int_{\Omega_{0}} h_{\imath}(\omega ; g) B_{i \jmath}(\omega) d \mu^{\left[g, f\left(\cdot, x_{i}\right)\right]} \\
& Z(\omega)\left(Y(\omega ; g)-\sum_{i, j=1}^{p} h_{\imath}(\omega ; g) B_{i \jmath}(\omega) x_{j},\right. \\
= & \left.\left.\sum_{i, j=1}^{p} h_{i}(\omega ; g) B_{\imath \jmath}(\omega) x_{j}\right)\right) \mu(d \omega)
\end{aligned}
$$

$=0$ 。

This implies our theorem.

The following is obvious from Lemma (4.15).

(5.7) Theorem. For each $\omega \in \Omega$ and $n \geqq 0$, let $\nu_{\omega}^{(n)}=\left(w_{i_{1}} \cdots w_{i_{n}}\right)^{-1} \chi_{\Omega_{\omega}^{(n) \nu}}$. Then the probability measure on $C([0, \infty) ; S)$ induced by $\left\{T^{n} w\left(\lambda^{-n} \cdot \wedge \sigma_{F_{n}}\right)\right\}$ under $P_{\mu_{\omega}^{(n)}}(d w)$ is the same as one induced by $\left\{w\left(\cdot \wedge \sigma_{F_{0}}\right)\right\}$ under $P_{\mu}(d w)$. Here $\sigma_{F_{n}}(w)$ $=\inf \left\{t \geqq 0 ; w(t) \in F_{n}\right\}$.

\section{§6. Examples}

Example 1. Let $D=N=M \geqq 3$ and $w_{1}=\cdots=w_{N}=\frac{1}{N}$. Let 


$$
a_{i \jmath}^{(k)}=\left\{\begin{array}{cl}
1 & i=j=k \\
0 & i=k, j \neq k \\
\frac{2}{N+2} & j=k, i \neq k \quad, i, j, k=1, \cdots, N . \\
\frac{2}{N+2} & i=j, i \neq k \\
\frac{1}{N+2} & i \neq j, i \neq k, j \neq k
\end{array}\right.
$$

Let $Q_{0}=Q_{1}=\left(\frac{1}{N-1}\right)^{1 / 2} I_{V_{0}}$ and $\lambda=\frac{1}{N+2}$, and let $J=\{\{(i, j),(j, i)\} ; i, j=1, \cdots$, $N, i \neq j\}$. Then all assumptions are verified. In this case the abstract topological space $S$ can be regarded as a fractal space so-called Sierpinski gasket. One can prove that the associated diffusion processes are essentially the same as ones constructed in $[1],[5],[7],[9]$ with reflecting boundary condition (see Shin [11]). Since one can show that $\left\|\wedge^{2} Y_{i}\right\|=N(N+2)^{-2}<\lambda, i=1, \cdots, N$, one can see that the index $p$ is equal to one. It is easy to check that $\nu \neq \mu$, and so we see by Corollary (2.12) that $\mu$ is ergodic and is singular relative to $\nu$. Thus the martingale dimension of our diffusion processes are one and the increasing process $\left\langle M^{1}, M^{1}\right\rangle_{t}$ given in Theorem (5.4) is a singular continuous

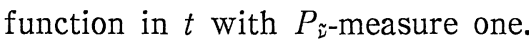

Example 2. Recently various diffusion processes on the nested fractal have been constructed by Lindstrøm [10]. Following his notions, we give the other examples. Let us take a nested fractal and fix it. Let $D=M$ be the number of the essential fixed points, and $N$ be the number of fixed points which is equal to the number of contractive similitudes $\left\{\phi_{1}, \cdots, \phi_{N}\right\}$. We may assume that the fixed point $x_{i}$ for $\phi_{i}, i=1, \cdots, D$, is an essential fixed point. Let $w_{1}=\cdots=w_{N}=\frac{1}{N}$, and let us define the equivalence relation $\sim$ on $\{1, \cdots, N\}$ $\times\{1, \cdots, D\} \backslash\{(i, i) ; i=1, \cdots, D\}$ by $(i, j) \sim\left(i^{\prime}, j^{\prime}\right)$ if $\phi_{\imath}\left(x_{j}\right)=\phi_{i^{\prime}}\left(x_{j^{\prime}}\right)$. We define $J$ from this equivalence relation.

Let us fix a good basic transition probability which is a fixed point given by Theorem V.5 in [10]. Then we have an associated homogeneous Markov chain on 1-points. Let $u^{i}, i=1, \cdots, D$, be the function on 1-points for which $u^{i}$ is harmonic relative to this associated Markov chain outside 0 -points and $u^{i}\left(x_{j}\right)$ $=\delta_{i j}, i, j=1, \cdots, D$. Now let us define $a_{i \jmath}^{(k)}, i, j=1, \cdots, D, k=1, \cdots, N$, by $a_{\imath \jmath}^{(k)}=u^{\jmath}\left(\psi_{k} x_{i}\right)$. Then by the results of [10], we can verify our assumptions and the abstract topological space $S$ can be regarded as the given nested fractal.

We conjecture that our diffusion process and the diffusion process given by Lindstrom [10] are the same and that the martingale dimension is one. 


\section{References}

[1] Barlow, M. T. and E. A. Perkins, Brownian motion on the Sierpinski gasket, Prob. Theo. Rel. Fields, 79 (1988), 543-624.

[2] Bougerol, P. and J. Lacroix, Products of random matrices with applications to Schrödinger operators, Progress in Prob. and Stat. vol. 8, Birkhäuser, Boston-BaselStuttgart, 1985.

[3] Bourbaki, N., Éléments de mathématique, Topologie générale, Hermann, Paris, 1965.

[4] Davis, M.H.A. and P. Varaiya, The multiplicity of an increasing family of $\sigma$-fields, Ann. Prob., 2 (1974), 958-963.

[5] Fukushima, M., Dirichlet forms and Markov processes, North-Holland, Amsterdam, 1980.

[6] Goldstein, S., Random walks and diffusions on fractals, Percolation theory and ergodic theory of infinite particle systems (Minneapolis, Minn., 1984-85), 121-129, IMA Vol. Math. Appl. 8, Springer, New York-Berlin, 1987.

[7] Hattori, K., T. Hattori and H. Watanabe, Gaussian fields theories on general networks and the spectral dimensions, Prog. Theor. Phys. Suppl., 92 (1987), 108-143.

[8] Kigami, J., A harmonic calculus on the Sirpinski spaces, Japan J. Appl. Math., 6 (1989), 259-290.

[9] Kusuoka, S., A diffusion process on a fractal, Probabilistic methods in Mathematical Physics, Proc. of Taniguchi International Symp. (Katata and Kyoto, 1985) ed. K. Ito and N. Ikeda, 251-274, Kinokuniya, Tokyo, 1987.

[10] Lindstrøm, T., Brownian motion on nested fractals, Preprint.

[11] Shin, T., Master Thesis, Univ. of Tokyo. 